\title{
IDENTIFICACIÓN DE MODOS DE TORSIÓN EMPLEANDO LA MEDICIÓN DE VIBRACIONES AMBIENTALES Y EL ANÁLISIS ESPECTRAL EN DOS EDIFICIOS DE CONCRETO REFORZADO
}

\author{
Richard Godfrey Montero ${ }^{(1)}$, Víctor Schmidt Díaz ${ }^{(2)}$, Luis C. Esquivel Salas ${ }^{(2)}$ y Yi Cheng Liu Kuan ${ }^{(3)}$
}

\section{RESUMEN}

En esta investigación se realizó un análisis espectral de la respuesta estructural de dos edificios de concreto reforzado, de $18 \mathrm{~m}$ y $56 \mathrm{~m}$ de altura y con distintos niveles de irregularidad en planta, al ser sometidos a vibraciones ambientales. Específicamente, se han usado el método básico (BFD) y de descomposición en el dominio de la frecuencia (FDD) sobre registros de vibración ambiental adquiridos mediante tres acelerómetros triaxiales para la identificación de las frecuencias y los modos naturales de vibración. Para cada edificio se identificaron al menos tres modos de vibración en un rango de frecuencias comprendido entre $1,0 \mathrm{~Hz}$ y $6,0 \mathrm{~Hz}$. Los modos con mayor densidad espectral fueron exhibidos entre las frecuencias de $1,0 \mathrm{~Hz}$ y $3,0 \mathrm{~Hz}$. De las formas modales, se evidenció que, entre más irregularidad en planta, mayor será la presencia de modos de torsión y el acoplamiento de estos con modos de flexión.

Palabras clave: análisis operacional modal; vibraciones ambientales; efecto de torsión; dinámica estructural

\section{IDENTIFICATION OF TORSIONAL MODES USING AMBIENT VIBRATIONS AND SPECTRAL ANALYSIS IN TWO REINFORCED CONCRETE STRUCTURES}

\begin{abstract}
In this investigation, a spectral analysis of the structural response of two reinforced concrete buildings, of $18 \mathrm{~m}$ and $56 \mathrm{~m}$ of height and with different plan regularity, subjected to ambient vibrations was done. Specifically, natural frequencies and modes of vibrations were determined through applying basic frequency domain (BFD) and frequency domain decomposition (FDD) methods to ambient vibration data acquired by three triaxial accelerometers. At least three vibration modes were identified for each building in a range of frequencies comprehended between $1,0 \mathrm{~Hz}$ and $6,0 \mathrm{~Hz}$. Modes with more spectral density were exhibited between frequencies of 1,0 Hz and 3,0 Hz. From mode shapes,
\end{abstract}

\footnotetext{
Artículo recibido el 13 de noviembre de 2019 y aprobado para su publicación el 31 de diciembre de 2020. Se aceptarán comentarios y/o discusiones hasta cinco meses después de su publicación.

(1) FSA Ingeniería y Arquitectura, C.P. 10108, Mata Redonda, San José, Costa Rica. E-mail: richard.godfrey@ucr.ac.cr

(2) Instituto de Investigaciones en Ingeniería, Universidad de Costa Rica, C.P. 11501, Montes de Oca, San José, Costa Rica. E-mail: victor.schmidt@ucr.ac.cr, luiscarlos.esquivel@ucr.ac.cr

(3) Escuela de Ingeniería Civil, Facultad de Ingeniería, Universidad de Costa Rica, C.P. 11501, Montes de Oca, San José, Costa Rica. E-mail: yi.liukuan@ucr.ac.cr
} 
it was determined that structures with more plan irregularity presents more torsional modes and coupling of these with flexural modes.

Keywords: operational modal analysis; ambient vibrations; torsional effect; structural dynamics

\section{INTRODUCCIÓN}

Las estructuras civiles a lo largo de su vida útil deberán resistir la acción de cargas gravitacionales y laterales. Estas últimas se relacionan con la respuesta dinámica de la estructura, y suelen distinguirse según su origen: viento, oleaje y sismo. En Costa Rica, debido a los sistemas estructurales típicos y al contexto geológico, la fuerza de sismo es la que generalmente suele regir en el diseño de las estructuras.

El daño estructural en edificios producto del movimiento sísmico ha sido relacionado con el efecto de torsión. Dicho efecto suele agravarse en aquellas estructuras con geometrías complejas y distribuciones irregulares de masa y rigidez en planta o en elevación (Ditomasso et al., 2010). La asimetría en la distribución de masa y rigidez en planta provoca que el centro de masa y el centro de rigidez no coincidan espacialmente. Como consecuencia de esto, ante la presencia de cargas laterales la estructura rota respecto a su centro de rigidez y ocurre un incremento significativo en las fuerzas resistidas por los componentes estructurales, especialmente en aquellos que están lejos de dicho punto. Esto último podría ocasionar la falla estructural de los mismos de no haber sido considerado durante el proceso del diseño.

Según diferentes investigaciones (Cherry y Brady, 1959; Chopra, 2007; Ditomasso et al., 2010), la respuesta de una estructura sujeta a cargas dinámicas puede ser descrita a partir de propiedades como la frecuencia natural de vibración, la forma y el amortiguamiento modales. Las anteriores pueden obtenerse experimentalmente por medio del análisis de registros de vibración en la estructura, y pueden ser utilizadas para la calibración de modelos matemáticos que predicen la respuesta estructural o en la evaluación del estado de salud de la estructura.

En edificaciones, principalmente, se distinguen tres tipos de vibraciones: sísmicas, forzadas y ambientales. Las vibraciones sísmicas son aquellas producidas por la ocurrencia de movimientos telúricos; las forzadas son el resultado de la excitación de la estructura mediante un sistema fijado a la misma que vibra a una determinada frecuencia; y las ambientales se asocian con el uso habitual de la estructura, la acción del viento, los microtremores, y el tránsito vehicular cercano (Murià y González, 1995). De las anteriores, las ambientales son las más sencillas y económicas de medir, pues no requieren de instrumentación permanente ni de mecanismos de excitación. Asimismo, a diferencia de las sísmicas y forzadas, para este tipo de vibración la estructura exhibe una respuesta elástica-lineal, por lo que el daño estructural es minimizado. No obstante, debido a la magnitud y a las fuentes de excitación de las vibraciones ambientales, éstas suelen ser más sensibles a la presencia del ruido. Sin embargo, se ha demostrado que los resultados obtenidos de las mismas son confiables (Yuen, Beck, y Katafygiotis, 2002).

Las propiedades dinámicas de un sistema estructural pueden ser estimadas mediante la aplicación de la teoría del análisis espectral sobre registros de vibración ambiental medidos en la estructura (Bendat y Piersol, 2010). A pesar de que dicha teoría se estableció para procesos estocásticos continuos e infinitos en el tiempo su aplicación sobre este tipo de datos es válida. Esto porque las amplitudes son aleatorias y las propiedades estadísticas se mantienen constantes en el tiempo; cumpliendo así con los criterios de estocasticidad, estacionariedad y ergodicidad (Henao, 2013).

Durante las últimas dos décadas, a nivel internacional la medición de vibraciones ambientales y la teoría del análisis espectral han sido ampliamente empleadas para la obtención de las propiedades dinámicas 
de estructuras. Sin embargo, en Costa Rica las investigaciones referentes al tema son recientes. Una de las primeras investigaciones fue desarrollada por Carvajal (2005), quien identificó las frecuencias naturales asociadas a modos de flexión en dos edificios de concreto reforzado mediante el uso de funciones de transferencia. Por su parte, en el Laboratorio Nacional de Materiales y Modelos Estructurales de la Universidad de Costa Rica (LanammeUCR), como parte del programa de monitoreo estructural de la Unidad de Puentes, se han dirigido varias investigaciones (Liu, 2015; Montalto, 2016; Garita, 2016; Irías, 2016) en las que se han identificado las propiedades dinámicas en puentes mediante los métodos de identificación de sistemas multivariables en el dominio del tiempo y descomposición en el dominio de la frecuencia (FDD). De manera similar a Carvajal (2005), Esquivel y Schmidt (2016) identificaron las propiedades dinámicas de tres edificios de concreto reforzado empleando el método básico en el dominio de la frecuencia (BFD). En este caso, además de determinar las frecuencias naturales asociadas a modos de flexión, se determinaron los amortiguamientos.

Actualmente, en Costa Rica el Laboratorio de Ingeniería Sísmica de la Universidad de Costa Rica (LIS-UCR) es la principal institución dedicada a la investigación de edificaciones mediante el uso de vibraciones ambientales. En el LIS-UCR, éstas han sido primordialmente empleadas para la identificación de las frecuencias naturales y el amortiguamiento modal de edificaciones asociadas a modos de flexión. Para la medición de vibraciones ambientales se ha utilizado un arreglo de instrumentación que consiste en la colocación de acelerómetros en varios niveles del edificio (uno por nivel). Mientras que para el análisis se ha hecho uso de los espectros de Fourier y de los cocientes espectrales (Esquivel y Schmidt, 2016). Los resultados obtenidos de estas prácticas resultan ser eficientes para la identificación de frecuencias naturales de modos de flexión, pero complejos o incompresibles para el caso de modos de torsión.

De acuerdo con los antecedentes, se ha concluido que la identificación de modos de torsión en edificios no ha sido explorada en Costa Rica. Debido a esto, en el presente trabajo de investigación se propone una metodología de instrumentación basada en investigaciones internacionales (Ditomasso et al., 2010; Henao, 2013; Brincker y Ventura, 2015) y el uso de la teoría del análisis espectral (Bendat y Piersol, 1966; Ewins, 1986; Brincker et al., 2001) para la identificación de las frecuencias naturales asociadas a modos de torsión en edificaciones. En este caso, se emplea el método de BFD y FFD para la obtención de las frecuencias naturales y formas modales en dos edificios de concreto reforzado con distintas clasificaciones de regularidad en planta según el Código Sísmico de Costa Rica 2010: uno regular en planta y el otro con irregularidad grave. Adicionalmente, se pretende evidenciar la relevancia de los modos de torsión según el nivel de irregularidad en planta de la estructura.

\section{MARCO TEÓRICO}

En esta investigación para la identificación de las propiedades dinámicas de los edificios se emplearon el método básico en el dominio de la frecuencia (BFD) y el método de descomposición en el dominio de la frecuencia (FDD). Ambos métodos están basados en la teoría del análisis espectral. Debido a lo anterior, en este apartado se presentan las principales consideraciones teóricas para dichos métodos.

\section{Teoría del análisis espectral}

De acuerdo con Bendat y Piersol (1966), el análisis espectral es un tipo de estimación no paramétrica que permite caracterizar un sistema lineal a partir de registros de entrada o salida. En el caso de estructuras, el análisis espectral permite estimar propiedades dinámicas como las frecuencias naturales de vibración, las formas y el amortiguamiento modales usando registros de vibración ambiental. 
Las propiedades dinámicas de la estructura son estimadas mediante la interpretación de las funciones de densidad espectral de los registros de vibración ambiental. Dichas funciones están definidas en el dominio de la frecuencia, y permiten visualizar la ocurrencia de la resonancia en el sistema estructural.

Las funciones de densidad espectral pueden ser estimadas como el resultado de la aplicación de la transformada de Fourier sobre las funciones de correlación. Si se consideran dos procesos estocásticos, estacionarios y ergódicos, $\{x(t)\}$ y $\{y(t)\}$, las funciones descritas están dadas por las siguientes expresiones matemáticas:

$$
\begin{aligned}
& R_{x y}(\tau)=\frac{1}{T} \int_{0}^{T} x(t) y(t+\tau) d t \\
& S_{x y}(f)=\int_{-\infty}^{\infty} R_{x y}(\tau) e^{-j 2 \pi f t} d \tau
\end{aligned}
$$

donde $R_{x y}(\tau)$ es la función de correlación cruzada y $S_{x y}(f)$ el espectro de densidad cruzado. Cuando los procesos $\{x(t)\}$ y $\{y(t)\}$ son idénticos, las funciones anteriores se convierten en la función de autocorrelación $R_{x x}(\tau)$ y en el autoespectro de densidad $S_{x x}(f)$ :

$$
\begin{aligned}
& R_{x x}(\tau)=\frac{1}{T} \int_{0}^{T} x(t) x(t+\tau) d t \\
& S_{x x}(f)=\int_{-\infty}^{\infty} R_{x x}(\tau) e^{-j 2 \pi f t} d \tau
\end{aligned}
$$

Mediante el autoespectro de densidad se visualiza la distribución de la energía o potencia con respecto a la frecuencia de una señal. En aquellas frecuencias donde se manifiesta la resonancia se dice que puede existir un modo de vibración. Asimismo, permite distinguir las frecuencias naturales propias del sistema del ruido en la señal. Por su parte, el espectro de densidad cruzado brinda información sobre la distribución de la energía compartida y el ángulo de fase relativo $(\theta)$ entre dos señales con respecto a la frecuencia. A partir de estas funciones, se determinan las frecuencias naturales y las formas modales del sistema estructural.

Las funciones de densidad espectral están definidas para todas las frecuencias, incluyendo valores tanto positivos como negativos, por lo que son espectros bilaterales. No obstante, estos son de mayor uso para estudios analíticos, en el caso de aplicaciones ingenieriles se prefiere usar los espectros unilaterales:

$$
\begin{aligned}
& G_{x y}(f)=\left\{\begin{array}{rr}
2 S_{x y}(f), & f \geq 0 \\
0, & f<0
\end{array}\right. \\
& G_{x x}(f)=\left\{\begin{array}{rr}
2 S_{x x}(f), & f \geq 0 \\
0, & f<0
\end{array}\right.
\end{aligned}
$$

El autoespectro de densidad y el espectro de densidad cruzado están relacionados mediante la función de coherencia que está dada por:

$$
\gamma_{x y}^{2}(f) \leq \frac{\left|G_{x y}(f)\right|^{2}}{G_{x x}(f) G_{y y}(f)}
$$


La función de coherencia determina la contribución de energía de una señal sobre otra. Un valor cercano a la unidad indica que las señales están altamente relacionadas, mientras que un valor de cero indica que no están relacionadas.

\section{Método básico en el dominio de la frecuencia $(B F D)$}

El método BFD fue propuesto por Bendat y Piersol (1993) y consiste en el uso de las funciones de densidad espectral para la identificación de frecuencias y modos naturales de vibración. En éste los parámetros modales son identificados mediante la selección de picos en las funciones de densidad espectral. Debido a lo anterior, popularmente también es conocido como el método de selección de picos.

Según Rainieri y Fabbrocino (2014), para la identificación de los parámetros modales, los autoespectros de densidad, espectros de densidad cruzados y la coherencia son calculados con respecto a un sensor de referencia. Las frecuencias naturales son identificadas como aquellas en las que ocurre la resonancia del sistema en los espectros de amplitud. Mientras que los modos de vibración son construidos a partir de las amplitudes y las fases de los espectros cruzados.

\section{Método de descomposición en el dominio de la frecuencia (FDD)}

El método FDD fue propuesto por Brincker, Zhang y Andersen (2001). Esta técnica se basa en la descomposición de valores singulares (SVD) de la matriz de densidad espectral (PSD) para la obtención de las frecuencias y los modos naturales de vibración.

Según Rainieri y Fabbrocino (2014), una de las demostraciones teóricas se basa en la expansión modal de la respuesta estructural $\{y(t)\}$ :

$$
\{y(t)\}=[\Phi]\{p(t)\}
$$

donde $[\Phi]$ es la matriz modal y $\{p(t)\}$ es la matriz de coordenadas modales.

De acuerdo con Vandiver (2011), la teoría de la expansión modal permite aproximar la respuesta estructural como una sumatoria de la contribución individual de cada uno de los modos naturales de vibración. La matriz modal [Ф] contiene los vectores singulares que representan cada una de las formas modales del sistema. Mientras que, el vector de coordenadas modales $\{p(t)\}$ contiene la contribución de cada una de las formas modales. expresión:

Seguidamente, si a la respuesta estructural se le aplica la función de correlación se obtiene la siguiente

$$
\left[R_{y y}(\tau)\right]=E\left[\{y(t+\tau)\}\{y(t)\}^{T}\right]=[\Phi]\left[R_{p p}(\tau)\right][\Phi]^{T}
$$

En la ecuación anterior, $\left[R_{y y}(\tau)\right]$ y $\left[R_{p p}(\tau)\right]$ son la matriz de correlación de la respuesta estructural y de las coordenadas modales. Mediante la aplicación de la transformada de Fourier sobre las matrices de correlación se obtienen las matrices de densidad espectral:

$$
\left[G_{y y}(f)\right]=[\Phi]\left[G_{p p}(f)\right][\Phi]^{T}
$$


donde $\left[G_{y y}(f)\right]$ y $\left[G_{p p}(f)\right]$ son la matriz de densidad espectral de la respuesta estructural y de las coordenadas modales. La matriz $\left[G_{p p}(f)\right]$ es diagonal cuando los modos de vibración no están correlacionados entre sí.

No obstante, en el caso de estructuras, debido a la dificultad de medir y controlar las fuentes de excitación, solamente se mide la respuesta estructural. Como consecuencia de esto, las matrices $\left[G_{p p}(f)\right]$ y [Ф] son desconocidas. Para solucionar lo anterior, se aplica la función de SVD sobre la matriz $\left[G_{y y}(f)\right]$ :

$$
\left[G_{y y}(f)\right]=[U][\Sigma][V]^{T}
$$

donde $[U]$ y $[V]$ son las matrices que contienen los vectores singulares y $[\Sigma]$ la matriz de valores singulares (ordenada de mayor a menor). Dado que la matriz $\left[G_{y y}(f)\right]$ puede considerarse como una matriz Hermitiana y positiva las matrices $[U]$ y $[V]$ son equivalentes, de manera que:

$$
\left[G_{y y}(f)\right]=[U][\Sigma][U]^{T}
$$

Comparando las dos expresiones para la matriz $\left[G_{y y}(f)\right]$ se tiene que:

$$
\begin{aligned}
& {\left[G_{y y}(f)\right]=[\Phi]\left[G_{p p}(f)\right][\Phi]^{T}=[U][\Sigma][U]^{T}} \\
& {[\Phi]=[U]} \\
& {\left[G_{p p}(f)\right]=[\Sigma]}
\end{aligned}
$$

De esta manera se encuentra que mediante la función SVD se puede estimar la matriz modal y la matriz de densidad espectral de las coordenadas modales.

En este método la interpretación de los resultados consiste en graficar la matriz de valores singulares en función de la frecuencia. De forma idéntica al método básico, los parámetros modales son determinados en aquellas frecuencias donde ocurre la resonancia del sistema. Seguido de esto, con la matriz de vectores singulares se visualizan las formas modales para cada una de las frecuencias naturales identificadas.

Dependiendo de la cantidad de sensores utilizados así será la dimensión de la matriz modal y de densidad espectral. Sin embargo, cuando existe un solo modo dominante, la matriz de densidad espectral de la respuesta puede expresarse como:

$$
\left[G_{y y}(f)\right]=\sigma_{1}\left\{u_{1}\right\}\left\{u_{1}\right\}^{T}
$$

donde $\sigma_{1}$ es el primer valor singular y $\left\{u_{1}\right\}$ la forma modal del modo dominante.

\section{CASOS DE ESTUDIO}

Seguidamente, se describen los sistemas estructurales de los dos edificios de concreto reforzado estudiados en esta investigación conforme a las disposiciones del Capítulo 4 del Código Sísmico de Costa Rica. 


\section{Edificio ED-1}

El edificio ED-1 es una estructura de cinco niveles de altura $(18,0 \mathrm{~m})$ utilizada para actividades con fines académicos (Figura 1). El sistema estructural ante cargas gravitacionales y laterales está constituido por marcos de concreto reforzado, y entrepisos de losas multitubulares pretensadas de $26 \mathrm{~cm}$ de espesor (incluyendo sobrelosa). Asimismo, el sistema de cimentación se compone de placas aisladas soportadas por medio de pilotes hincados.

Con respecto al sistema estructural, los elementos verticales tienen continuidad a lo largo de toda la elevación y están dispuestos de manera uniforme y simétrica respecto a la planta. Además, la forma de la planta es rectangular, por lo que el centro de masa y rigidez coinciden espacialmente con el centro geométrico de la misma. De acuerdo con estas características, la estructura es clasificada como regular tanto en altura como en planta. Asimismo, debido a la orientación de las columnas el eje de resistencia más fuerte coincide con el sentido corto de la planta estructural.

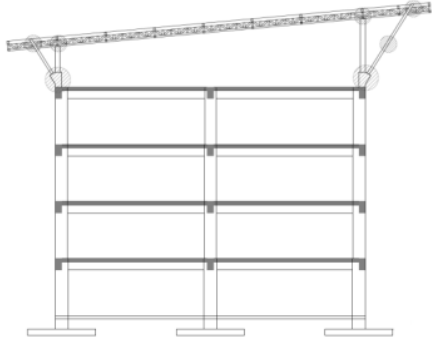

(a) Vista Lateral



(b) Vista en planta



Figura 1. Sistema estructural del edificio ED-1 visto en (a) elevación y (b) planta

\section{Edificio ED-2}

El edificio ED-2 es una torre de oficinas de 16 niveles de altura $(56,0 \mathrm{~m})$. El sistema estructural es de tipo dual y está constituido por marcos y muros de concreto reforzado conectados mediante entrepisos de viguetas pretensadas con un espesor de $26 \mathrm{~cm}$ (incluyendo sobrelosa). Los componentes verticales están cimentados mediante placas corridas y placas aisladas unidas mediante vigas de amarre.

Esta edificación se caracteriza por su diseño arquitectónico en forma piramidal y por tener únicamente un eje de simetría a $45^{\circ}$ respecto a la horizontal (Figura 2). De acuerdo con la configuración estructural, el centro de rigidez está situado en el núcleo de ascensores y escaleras, pues es donde se concentran los muros estructurales. Mientras que, el centro de masa se desplaza hacia el centro de rigidez conforme la planta disminuye sus dimensiones. En este caso, la excentricidad en planta excede el $25 \%$ por lo que ha sido clasificada como irregular grave.

En elevación los elementos verticales tienen continuidad en toda la altura de la edificación, y no hay cambios significativos de masa ni rigidez entre niveles, por lo que la estructura se ha clasificado como regular en altura. 


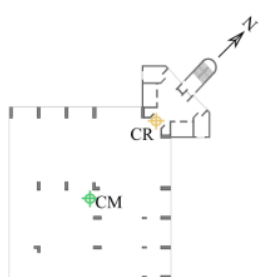

(a) Nivel de Sótano S2

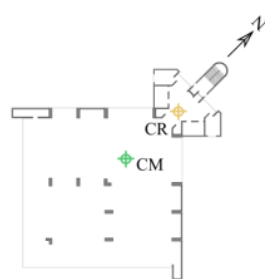

(b) Nivel 6



(c) Nivel 12



(d) Vista lateral

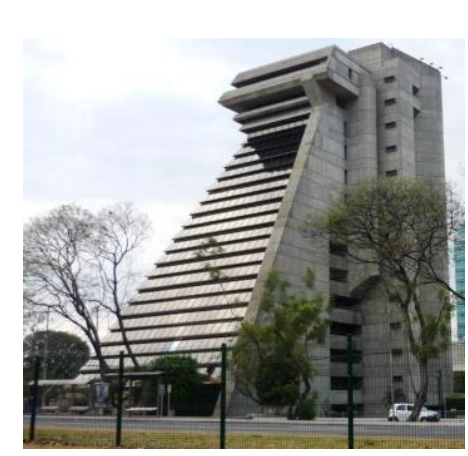

Figura 2. Plantas estructurales de los niveles (a) de sótano S, (b) N6 y (c) N12, y (d) vista lateral del edificio ED-2

Tanto en el edificio ED-1 como ED-2, los entrepisos fueron considerados como diafragmas rígidos. Esto porque los espesores y las relaciones de aspecto de las losas permiten suponer que estos son infinitamente rígidos en su plano.

\section{PROCEDIMIENTO DE INSTRUMENTACIÓN Y DE ANÁLISIS ESPECTRAL}

A continuación, se presenta el procedimiento empleado para la medición y el tratamiento de las vibraciones ambientales en las edificaciones estudiadas. Asimismo, se describe el procedimiento para la aplicación del método básico en el dominio de la frecuencia (BFD) y de descomposición en el dominio de la frecuencia (FDD) para la obtención de las frecuencias naturales y modos de vibración de las edificaciones.

\section{Mediciones de vibraciones ambientales}

Las mediciones de vibraciones ambientales de los edificios fueron realizadas mediante tres acelerómetros triaxiales de la marca Guralp facilitados por el LIS-UCR (Figura 3). Estos equipos tienen una escala sensitiva completa de $\pm 2,0 \mathrm{~g}$ y una frecuencia de muestreo de $200 \mathrm{~Hz}$. Además, cuentan con un cable de red para la extracción de los datos, una fuente de alimentación y un dispositivo de GPS.
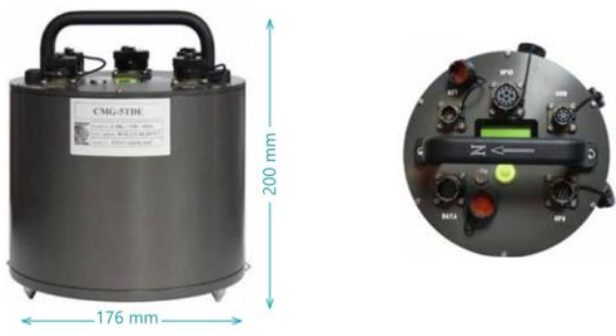

Figura 3. Acelerómetro Guralp CMG 5TDE. Fuente: Guralp Systems (2012)

Con los tres acelerómetros se registran nueve grados de libertad de la estructura: seis traslacionales y tres verticales. Como en esta investigación solamente se requieren estudiar modos de flexión y de torsión, 
únicamente se requieren los registros de los grados de libertad traslacionales. De acuerdo con Brincker y Ventura (2015), se requieren de al menos seis sensores para ejecutar adecuadamente el análisis operacional modal de una edificación.

En este caso, para la identificación de los parámetros dinámicos se ha propuesto usar dos tipos de configuraciones de los instrumentos que se denominaron como: prueba traslacional y prueba rotacional. En la prueba traslacional se colocó un acelerómetro en la base, uno en un piso intermedio y otro cercano a la azotea, todos sobre un mismo eje vertical cercano al centro de rigidez. Mientras que, en la prueba rotacional, en una misma planta se ubicó un acelerómetro sobre el centro de rigidez y los otros dos en extremos opuestos de la planta. Mediante la primera configuración es posible capturar los modos de flexión, y con la segunda los de torsión. De la Figura 4 y Figura 5 se pueden observar las configuraciones descritas para los dos edificios estudiados.

Para cada prueba se definió una ventana de medición de $20 \mathrm{~min}$, la cual contiene una cantidad total de 240,001 datos. Según Bendat y Piersol (2010), entre mayor la duración de la misma, menores serán los errores en las estimaciones. Asimismo, debido a la aleatoriedad de las fuentes de excitación se puede asegurar capturar una mayor cantidad de modos de vibración que podrían no ser manifestados en mediciones de corta duración.

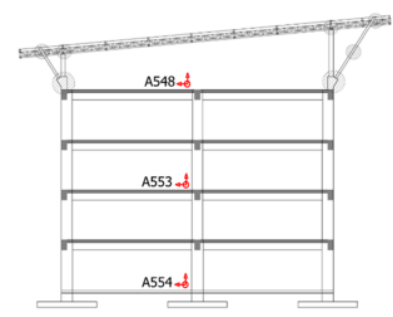

(a) Prueba traslacional



(b) Prueba rotacional

Figura 4. Configuración de los instrumentos para prueba (a) traslacional y (b) rotacional del edficio ED-1 
(a) Prueba traslacional



(b) Prueba rotacional



Figura 5. Configuración de los instrumentos para prueba (a) traslacional y (b) rotacional del edificio ED-2

\section{Tratamiento de los registros de vibración}

Los registros de vibración ambiental adquiridos mediante la prueba traslacional y rotacional fueron tratadas previo a la aplicación del análisis espectral por medio de rutinas programadas en el entorno de LINUX y que son utilizadas por el LIS-UCR (Esquivel y Schmidt, 2016). En términos generales, el tratamiento de los registros de vibración consiste en la asignación de unidades de aceleración, el cortado para la definición de las ventanas de análisis, la corrección por línea base y el filtrado de los datos.

En esta investigación los instrumentos de medición empleados registran los datos en unidades de cuentas de voltaje, por lo que se aplicaron factores de conversión para dar unidades de aceleración a los mismos. Asimismo, como las pruebas de vibración ambiental fueron ejecutadas de forma consecutiva, se cortó el registro total de datos para extraer la información correspondiente de las ventanas de análisis establecidas para cada prueba. Cada una de las ventanas resultantes se corrigieron por línea base para eliminar cualquier tendencia lineal, y se les aplicó un filtro de tipo pasabanda entre las frecuencias de 0,05 $\mathrm{Hz} \mathrm{y} 50,00 \mathrm{~Hz}$. Es importante destacar, que dicho filtro se caracteriza por ser de dos pasadas, lo cual permite reducir errores en la estimación de la fase relativa entre señales.

\section{Procedimiento de análisis espectral}

En el caso de esta investigación, el análisis espectral se aplicó sobre los registros de vibración ambiental adquiridos para cada tipo de prueba (traslacional y rotacional). De cada una de las señales registradas se estiman las funciones de densidad espectral y de coherencia por medio del programa computacional MATLAB (The Math Works, Inc, 2014). Para la estimación de las funciones anteriores se divide el registro total en ventanas de 8192 datos ( $40 \mathrm{~s}$, aproximadamente) y se acotan mediante la función de Hanning para evitar la fuga de la densidad espectral en las frecuencias bajas. Los valores estimados para 
cada una de las ventanas son promediados entre sí para obtener las amplitudes en el rango de frecuencias en cuestión. Los resultados obtenidos sirven como base para el estudio de la estructura mediante los métodos BFD y FFD.

\section{Método básico en el dominio de la frecuencia $(B F D)$}

Con los registros de la prueba traslacional, se utilizaron los autoespectros en las dos direcciones de análisis para identificar preliminarmente las frecuencias naturales asociadas con modos de flexión. Un modo de flexión se reconoce como puro en una dirección cuando la resonancia solamente ocurre en una de las direcciones. Seguidamente, se estudiaron la fase de los espectros cruzados y la coherencia entre señales de distintos niveles para determinar el tipo de modo correspondiente.

Mediante la prueba rotacional, se distinguen los modos de flexión de los de torsión. En este caso, se identifica un modo de flexión en aquella frecuencia en la que los autoespectros y espectros cruzados existe la resonancia del sistema, la coherencia es alta y el ángulo de fase es cero para todos los sensores. Mientras que, el modo de torsión se evidencia cuando los autoespectros de los sensores extremos presentan resonancia y el sensor del centro de rigidez no. En este caso, el ángulo de fase entre los sensores extremos es de $180^{\circ}$, indicando así un movimiento opuesto. Adicionalmente, la coherencia entre el sensor en el centro de rigidez y otro en el extremo muestra un valor próximo a cero, dado que no hay relación entre sus desplazamientos.

En el caso de acoplamiento entre modos de flexión y torsión, cuando predomina la flexión las amplitudes máxima y mínima coinciden con los sensores en los extremos de la planta, mientras que, en el caso contrario el centro de rigidez tiene la menor amplitud, y uno de los extremos tiene más amplitud que el otro.

Este método asume que alrededor de la resonancia únicamente existe un modo natural de vibración. Debido a esto, la técnica produce resultados aceptables cuando la estructura es ligeramente amortiguada y los modos de vibración están bien separados (Brincker, Zhang, y Andersen, 2001). Cuando existen modos naturales de vibración cercanos, el método lo que brinda es una deformada operacional que representa la superposición de las formas modales (Peeters y De Roeck, 2001). Asimismo, este método no es recomendado para estimar el amortiguamiento modal.

\section{Método descomposición en el dominio de la frecuencia (FFD)}

En el método FFD se construye la matriz de densidad espectral que es descompuesta para obtener los valores y vectores singulares de la respuesta de la estructura. En este caso, los valores singulares indican las frecuencias naturales, mientras que, los vectores singulares definen las formas modales. Para la identificación del modo de vibración se grafica en un plano los vectores singulares, y verificando las amplitudes y fase relativa entre sensores se determina el tipo de movimiento exhibido por la estructura.

\section{DISCUSIÓN DE RESULTADOS}

\section{Edificio ED-1}

En esta sección se discuten los resultados obtenidos mediante el método BFD y FDD sobre los registros de vibración ambiental adquiridos de las pruebas traslacional (Figura 6) y rotacional (Figura 7) en el edificio ED-1. 



(a) Componente E-O

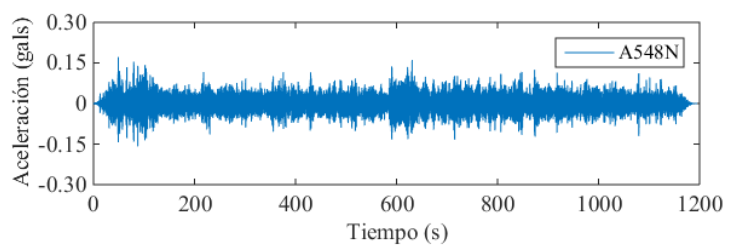

(b) Componente N-S

Figura 6. Registros de vibración ambiental para prueba traslacional del edificio ED-1
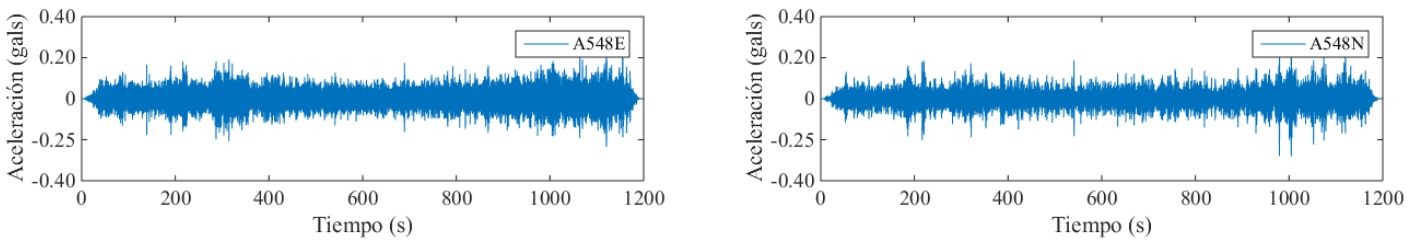

E1
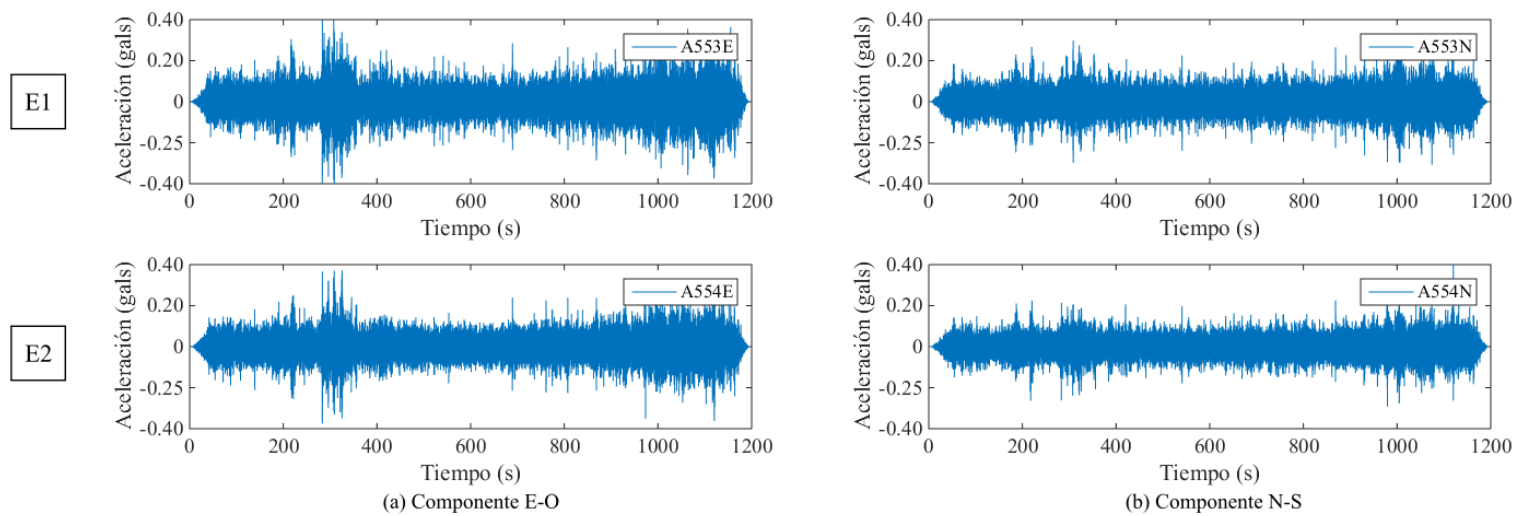

Figura 7. Registros de vibración ambiental para prueba rotacional en nivel N5 del edificio ED-1

\section{Método básico en el dominio de la frecuencia (BFD)}

En primera instancia, de los registros de vibración ambiental de la prueba traslacional, se estimaron los autoespectros en las direcciones E-O y N-S mostrados en la Figura 8. De acuerdo con estos, se identificó que en las frecuencias de $2,881 \mathrm{~Hz}, 3,564 \mathrm{~Hz}$ y $5,347 \mathrm{~Hz}$ ocurre la resonancia del sistema estructural. En 
todas ellas, la magnitud del autoespectro es proporcional a la elevación. Lo anterior resulta congruente, pues las máximas aceleraciones fueron registradas en el nivel N5.
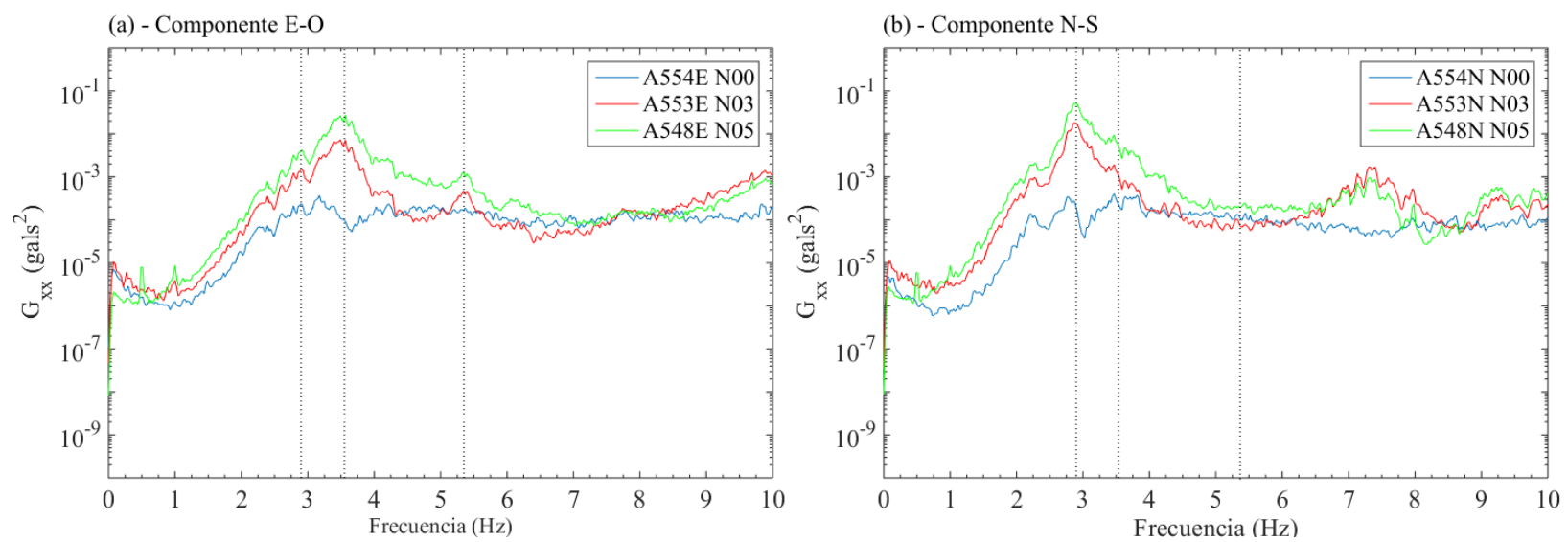

Figura 8. Autoespectros en dirección (a) E-O y (b) N-S de prueba traslacional en edificio ED-1

Según la amplitud de los autoespectros, la frecuencia natural de 2,881 Hz predomina en la dirección $\mathrm{N}-\mathrm{S}$, mientras que, en $3,564 \mathrm{~Hz}$ y $5,347 \mathrm{~Hz}$ predomina en la dirección E-O. No obstante, debido a la regularidad del sistema estructural, estas frecuencias han sido asociadas con modos de flexión. Para verificar la dirección de estos modos, se estimaron las funciones de coherencia mostradas en la Figura 9. En estas funciones se compararon dos sensores ortogonales entre sí en los niveles N3 y N5 de la estructura. Los resultados indican que la coherencia entre dichos sensores es baja, de manera que, los modos asociados tienen una dirección predominante.


Figura 9. Función de coherencia entre sensores ortogonales en (a) nivel 3 y (b) nivel 5 en edificio ED-1

En la Figura 10 se presentan los espectros de fase entre sensores paralelos en el nivel N3 y N5. En este caso, se determinó que en las frecuencias naturales de $2,881 \mathrm{~Hz}, 3,564 \mathrm{~Hz}$ y $5,347 \mathrm{~Hz}$ los sensores se encuentran en fase. 

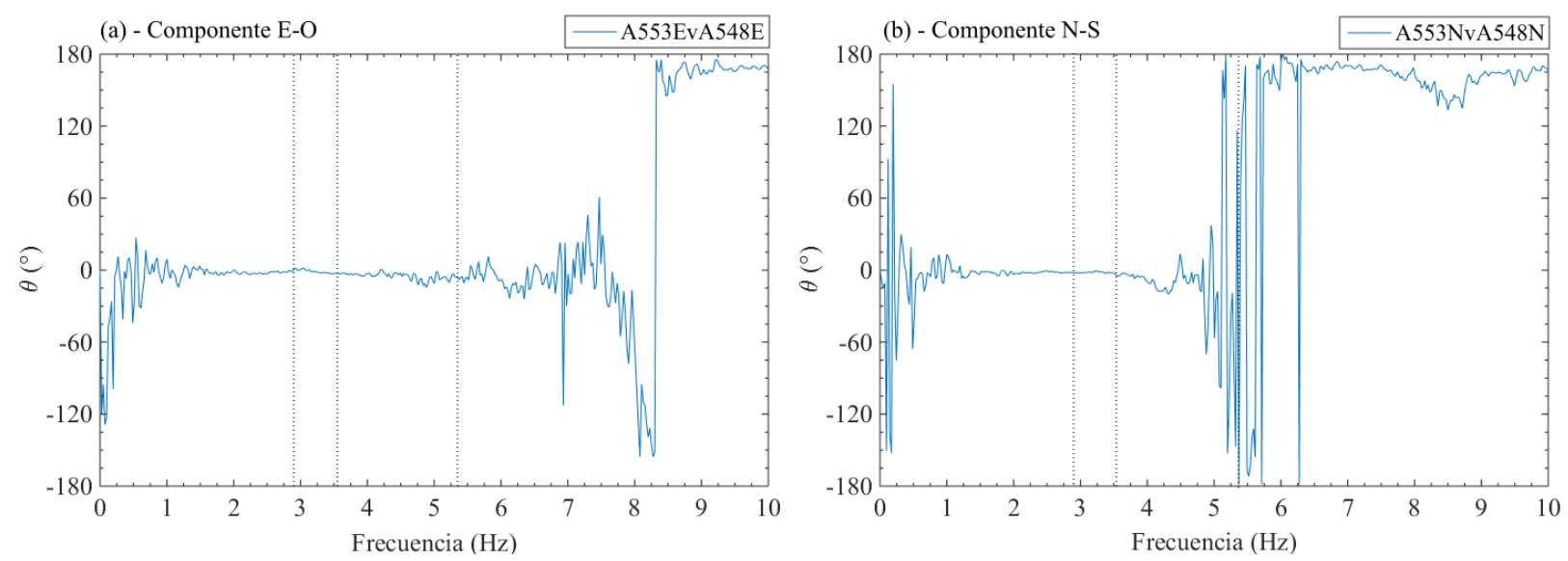

Figura 10. Espectro de fase entre sensores paralelos en nivel N3 y N5 en edificio ED-1

Seguidamente, se estimaron las funciones de densidad espectral y de coherencia de los registros de vibración ambiental de la prueba rotacional en el nivel N5. Esto para verificar los modos de flexión identificados e identificar posibles modos de torsión de la estructura.

De acuerdo con los autoespectros (Figura 11), las frecuencias naturales de 2,881 Hz, 3,564 Hz y 5,347 $\mathrm{Hz}$ vuelven a ser manifestadas. Las amplitudes de estos reflejan nuevamente una dirección predominante. No obstante, en ninguna de estas direcciones los sensores tienen la misma amplitud, por lo que existe un acoplamiento con un posible modo de torsión. Lo anterior es de esperar dado que ninguna estructura es perfectamente regular. A pesar de esto, en las frecuencias naturales de 2,881 Hz y 3,564 Hz la diferencia entre amplitudes en la dirección predominante es despreciable, mientras que, las amplitudes en la dirección ortogonal son bajas. Por tanto, los modos asociados con estas dos frecuencias pueden ser considerados como de flexión en una dirección. A diferencia de éstas, en las frecuencias naturales de 3,391 Hz y 5,347 Hz, se evidencia una diferencia significativa en ambas direcciones entre las amplitudes de los sensores centrales y extremos, por lo que se puede inferir que estas podrían estar relacionadas a un modo de torsión.


Figura 11. Autoespectros en dirección (a) E-O y (b) N-S de prueba rotacional en edificio ED-1

Para verificar que las frecuencias naturales de $3,931 \mathrm{~Hz}$ y $5,347 \mathrm{~Hz}$ están asociadas con modos de torsión, se han estimado los autoespectros mostrados en la Figura 12. En este caso, los registros utilizados corresponden a la resta entre dos señales paralelas ubicadas en los extremos de la planta. De esta manera, se reduce el contenido espectral asociado con los modos de flexión. Los resultados indican que en 3,931 Hz 
y $5,347 \mathrm{~Hz}$ ocurre la resonancia del sistema estructural, por lo que los modos son de torsión. Contrariamente, en las frecuencias naturales de $2,881 \mathrm{~Hz}$ y $3,564 \mathrm{~Hz}$ la amplitud del autoespectro es despreciable, respaldando así el supuesto de que estas corresponden a modos de flexión.

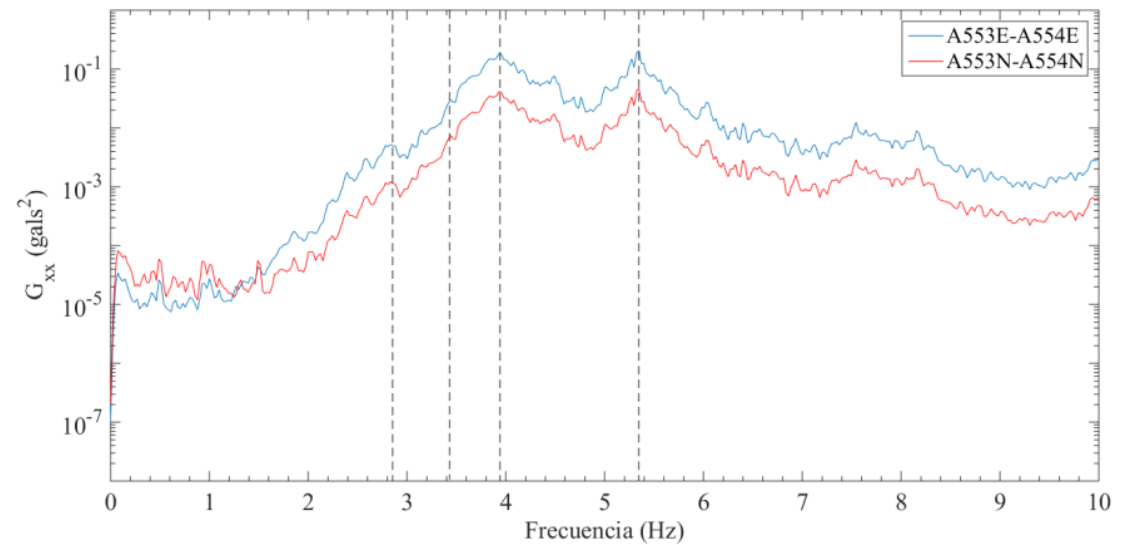

Figura 12. Autoespectros de señales sustraídas de prueba rotacional en edificio ED-1

Posteriormente, para validar los tipos de modos identificados se han usado los espectros de fase (Figura 13) y las funciones de coherencia (Figura 14) de la prueba rotacional. En las frecuencias naturales de $2,881 \mathrm{~Hz}$ y $3,564 \mathrm{~Hz}$ se observó que los sensores paralelos a la dirección predominante se encuentran en fase y presentan alta coherencia entre sí, reflejando así un movimiento traslacional de la planta. En el caso de la frecuencia natural de $5,347 \mathrm{~Hz}$, para las dos direcciones de análisis, los sensores paralelos situados en los extremos (E1-E2) presentan una alta coherencia y un ángulo de fase próximo a los $180^{\circ}$, mientras que, se obtuvieron valores bajos de coherencia entre el centro de rigidez y los extremos (CR-E1 y CR-E2). No obstante, en la frecuencia natural de $3,931 \mathrm{~Hz}$, se manifiesta el desfase entre los extremos, sin embargo, la coherencia es baja. De acuerdo con las dos frecuencias naturales anteriores, solamente la primera se puede evidenciar el movimiento rotacional de la planta.
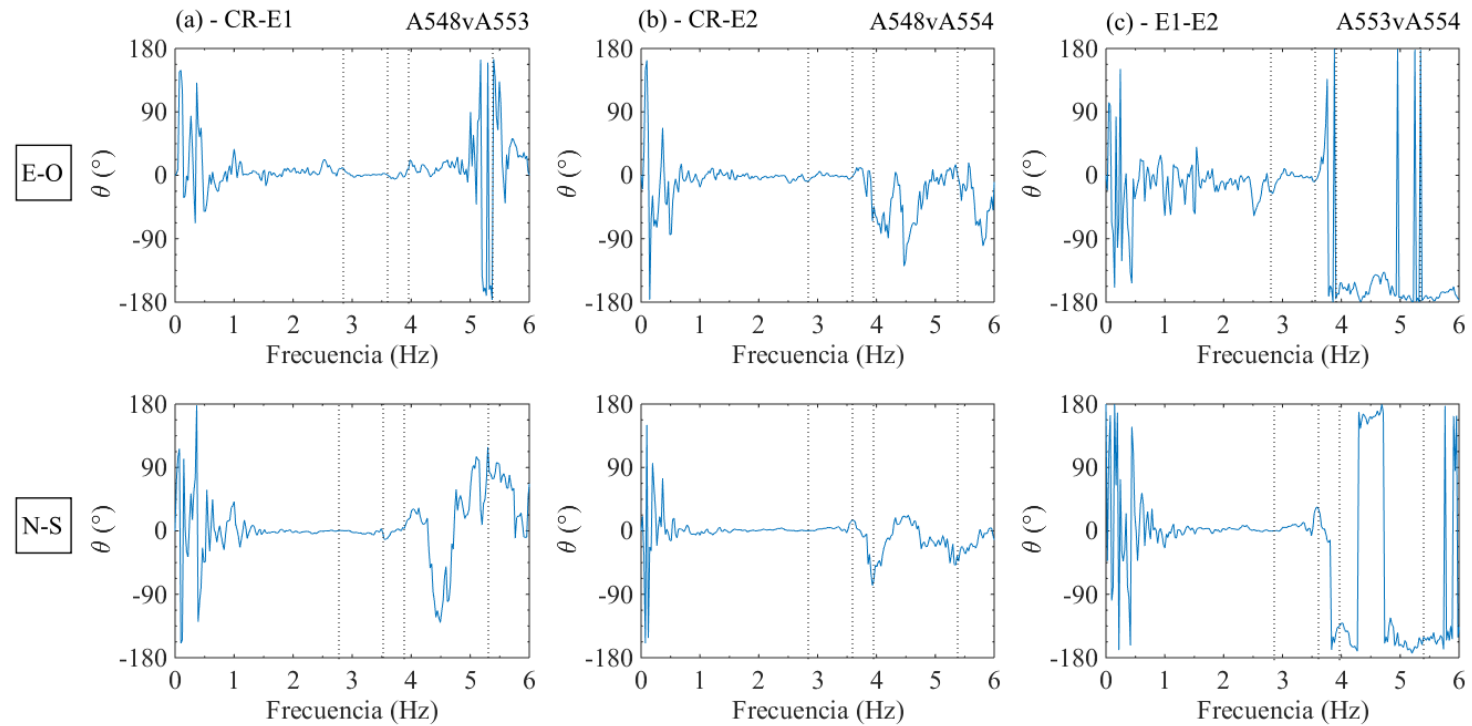

Figura 13. Espectros de fase entre señales (a) en centro de rigidez y un extremo, (b) centro de rigidez y otro extremo, y (c) extremos del edificio ED-1 

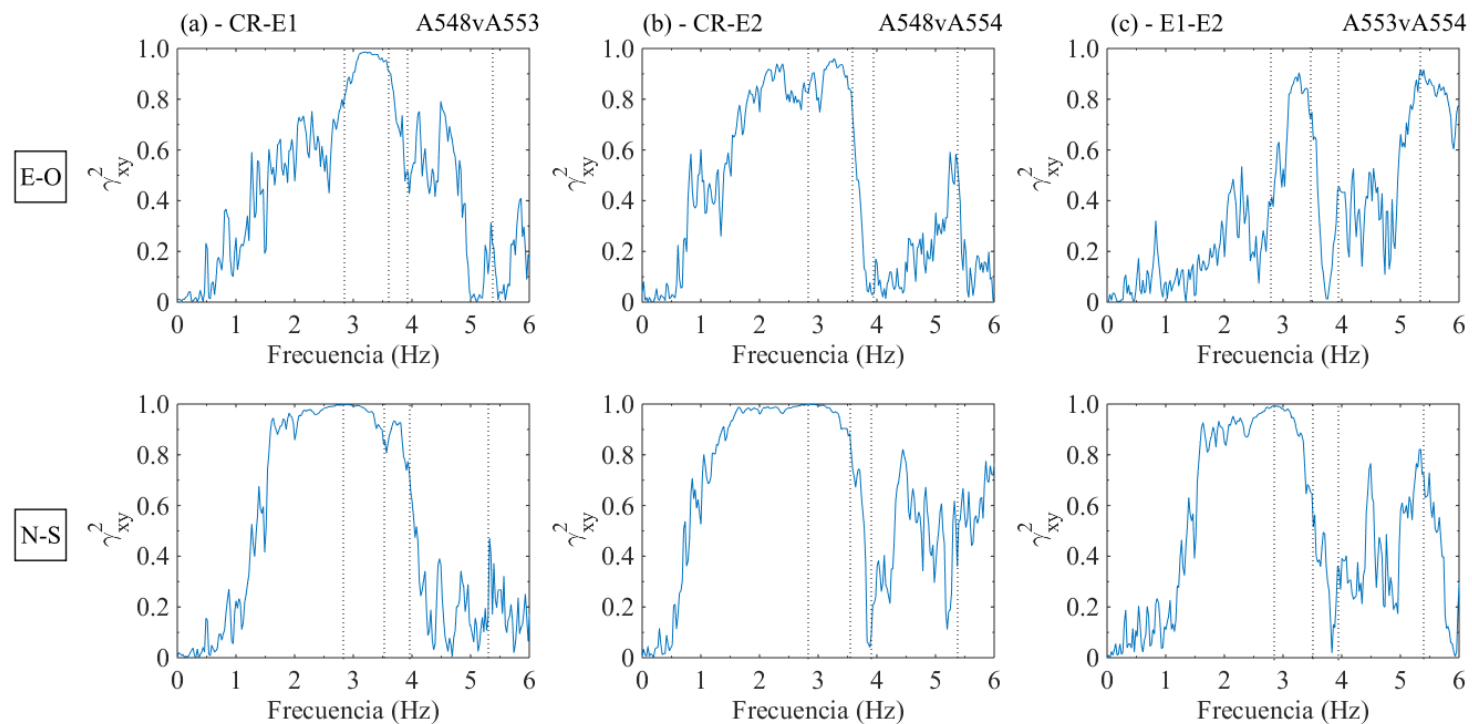

Figura 14. Coherencia entre señales en (a) centro de rigidez y un extremo, (b) centro de rigidez y otro extremo, y (c) extremos del edificio ED-1

A partir del método BFD, se determinó que las frecuencias de 2,881 Hz y 3,564 Hz corresponden a los modos de flexión en las direcciones N-S y E-O, respectivamente. Mientras que la frecuencia de 5,347 Hz está asociadas a un modo de torsión.

\section{Método de descomposición en el dominio de la frecuencia (FDD)}

A partir de las funciones de densidad espectral estimadas con el método BFD, se estimaron los valores singulares mediante el método FDD para cada una de las pruebas. En la Figura 15 se presenta el primer valor singular para la prueba traslacional y rotacional, respectivamente. Según estos resultados, la resonancia del sistema estructural ocurre para las frecuencias de $2,881 \mathrm{~Hz}, 3,564 \mathrm{~Hz}, 3,931 \mathrm{~Hz}$ y $5,347 \mathrm{~Hz}$.

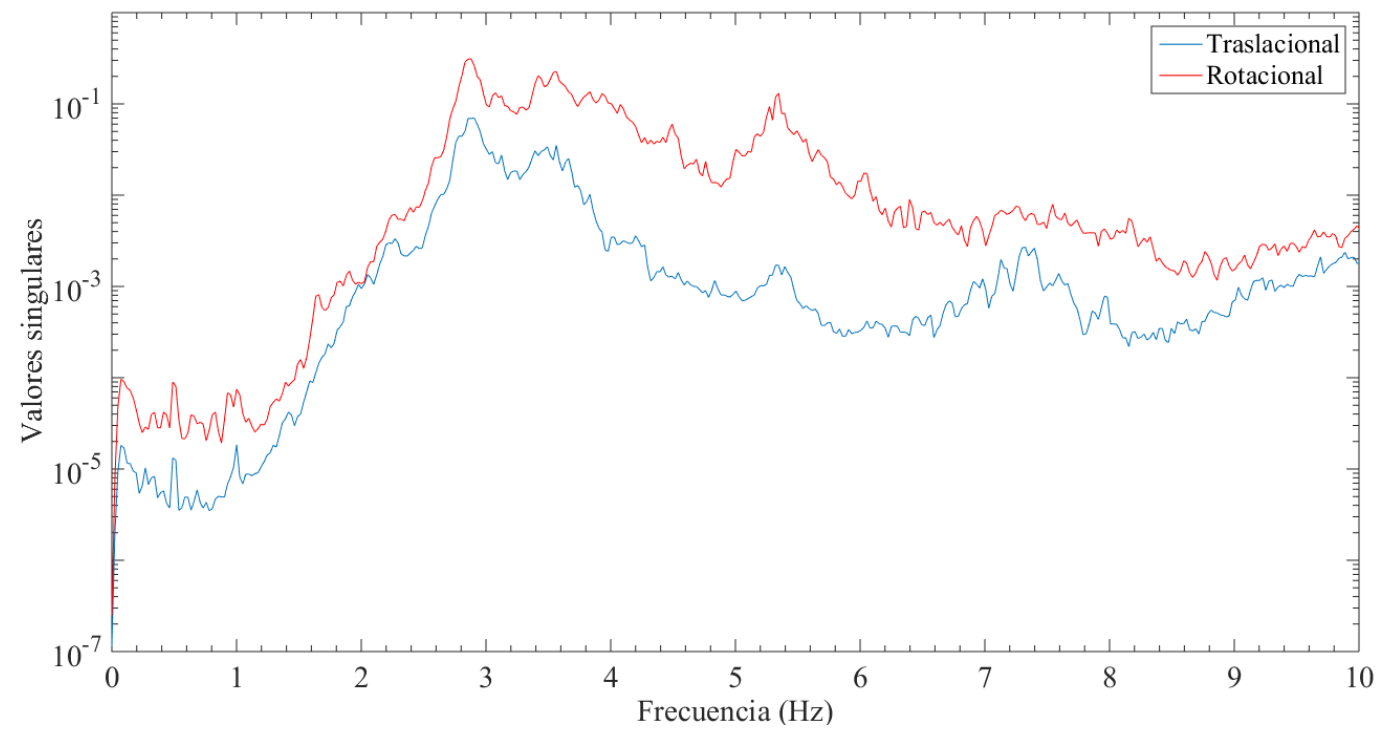

Figura 15. Valores singulares de la prueba (a) traslacional y (b) rotacional del edificio ED-1 
Para determinar el tipo de modo correspondiente se emplean los vectores singulares. En la Figura 16 presentan los vectores singulares de las frecuencias naturales de $2,881 \mathrm{~Hz}$ y $3,564 \mathrm{~Hz}$. Según las amplitudes de los vectores se identifica que existe una dirección predominante para cada una de estas. Por su parte, la fase relativa entre sensores de diferentes niveles evidencia que estos se encuentran en fase. Asimismo, esto ocurre para sensores en el nivel N5. De estos resultados, se ha determinado que las frecuencias naturales de 2,881 Hz y 3,564 Hz corresponden a modos de flexión en la dirección N-S y E-O, respectivamente.
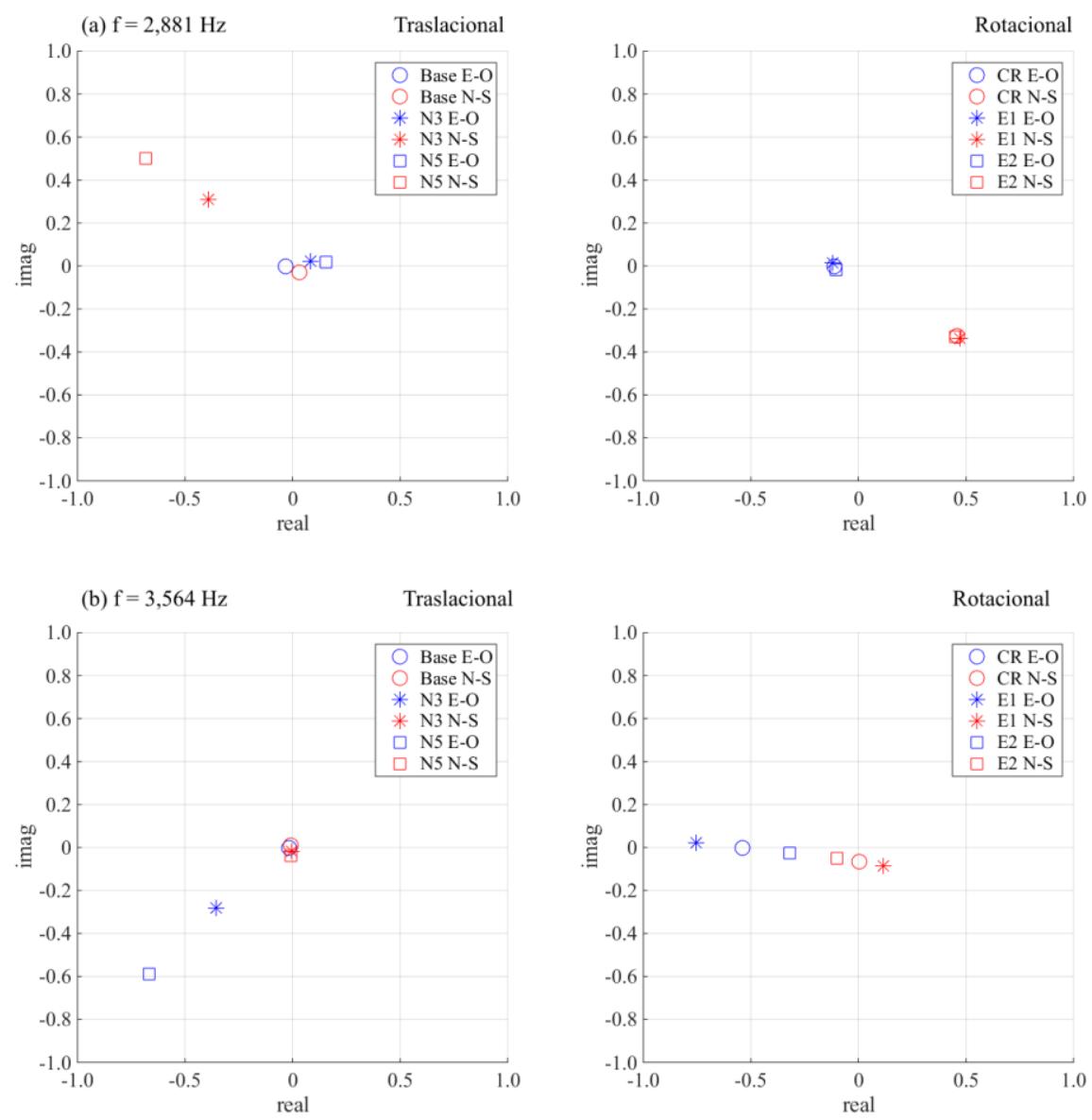

Figura 16. Vectores singulares de las frecuencias naturales de (a) 2,881 Hz y (b) 3,564 Hz en Edificio ED-1

No obstante, en la Figura 17 se muestran los vectores singulares de las frecuencias naturales de 3,931 $\mathrm{Hz}$ y $5,347 \mathrm{~Hz}$. De acuerdo con estos resultados, se ha determinado que existe un ángulo de fase de aproximadamente $180^{\circ}$ entre señales paralelas en los extremos de la planta. Sin embargo, solamente para la frecuencia de 5,347 Hz se evidencia que las amplitudes en el centro de rigidez son bajas en comparación a las de los extremos. Por tanto, nuevamente, se identifica que solo la frecuencia natural de 5,347 Hz corresponde a un modo de torsión.

\section{Formas modales}

De acuerdo con los resultados obtenidos de los métodos BFD y FDD se identificaron dos modos de flexión y dos de torsión. Las formas modales pueden ser observadas en la Figura 18. 

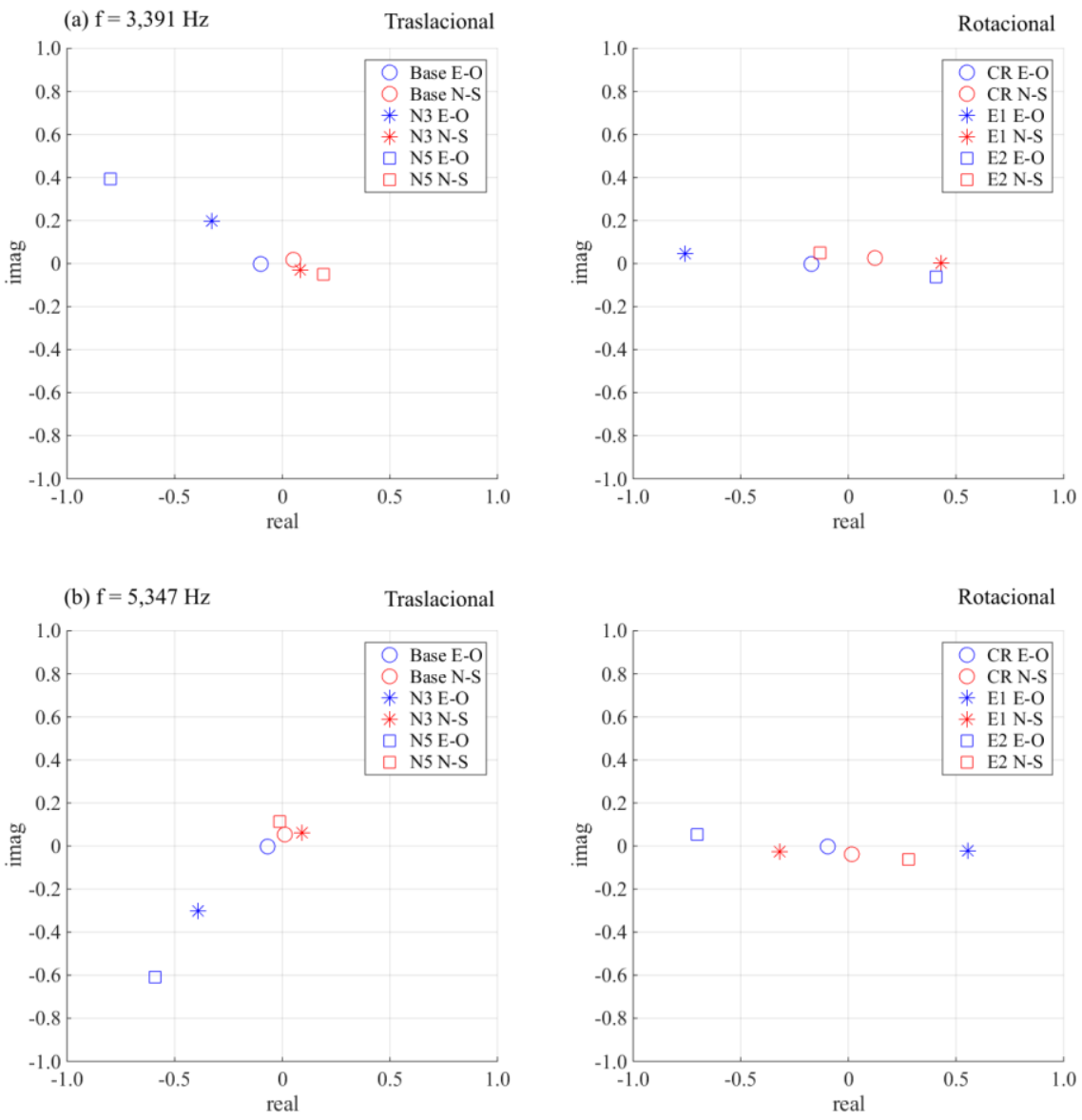

Figura 17. Vectores singulares de las frecuencias naturales de (a) 3,931 Hz y (b) 5,347 Hz en Edificio ED-1

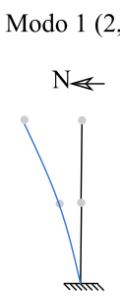

(a) Dirección N-S

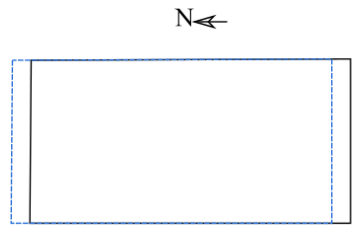

(b) Planta Nivel 5
Modo $2(3,564 \mathrm{~Hz})$

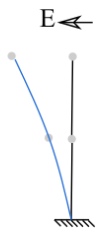

(a) Dirección E-O

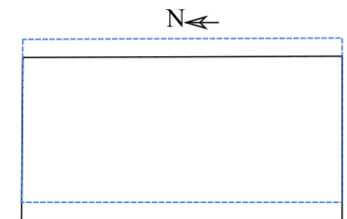

(b) Planta Nivel 5

Modo $3(5,347 \mathrm{~Hz})$

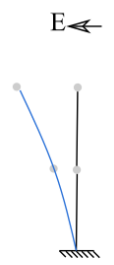

(a) Dirección E-O

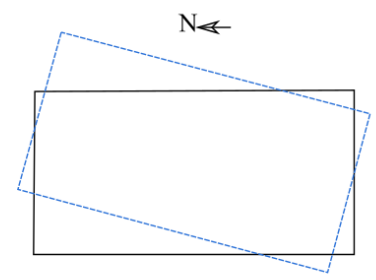

(b) Planta Nivel 5

Figura 18. Formas modales identificadas para edificio ED-1 


\section{Edificio ED-2}

A continuación, se presenta la discusión de los resultados obtenidos de los registros de vibración ambiental requeridos en las pruebas traslacional y rotacional del Edificio ED-2.
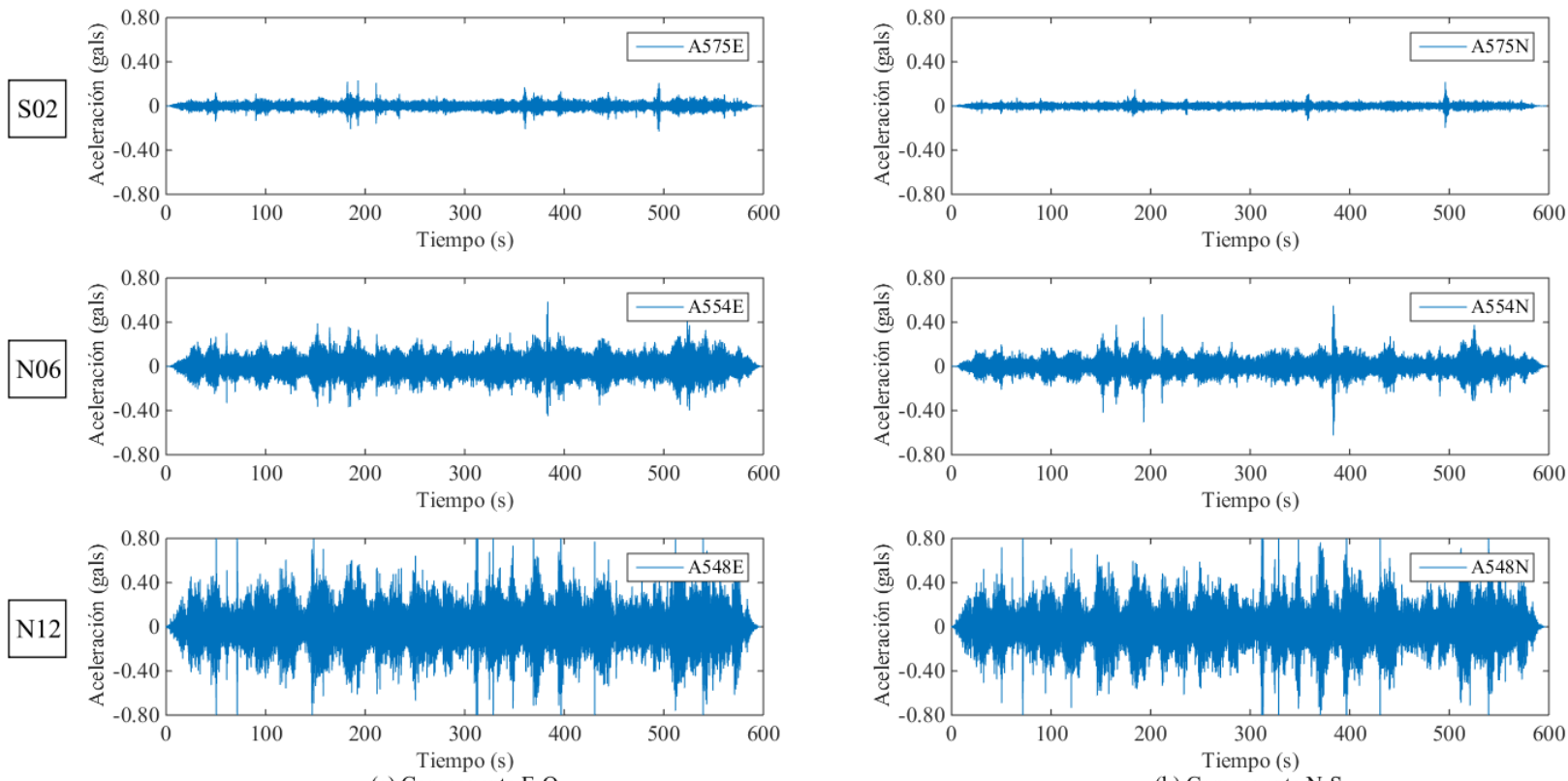

(a) Componente E-O

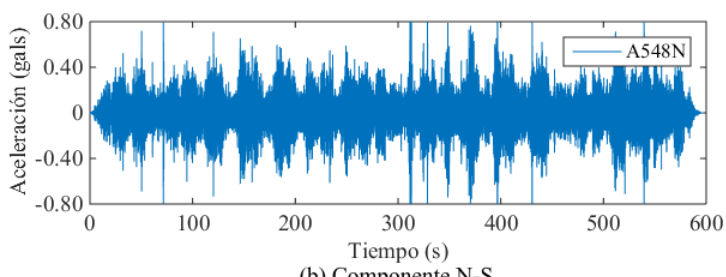

(b) Componente N-S

Figura 19. Registros de vibración ambiental para prueba traslacional del edificio ED-2
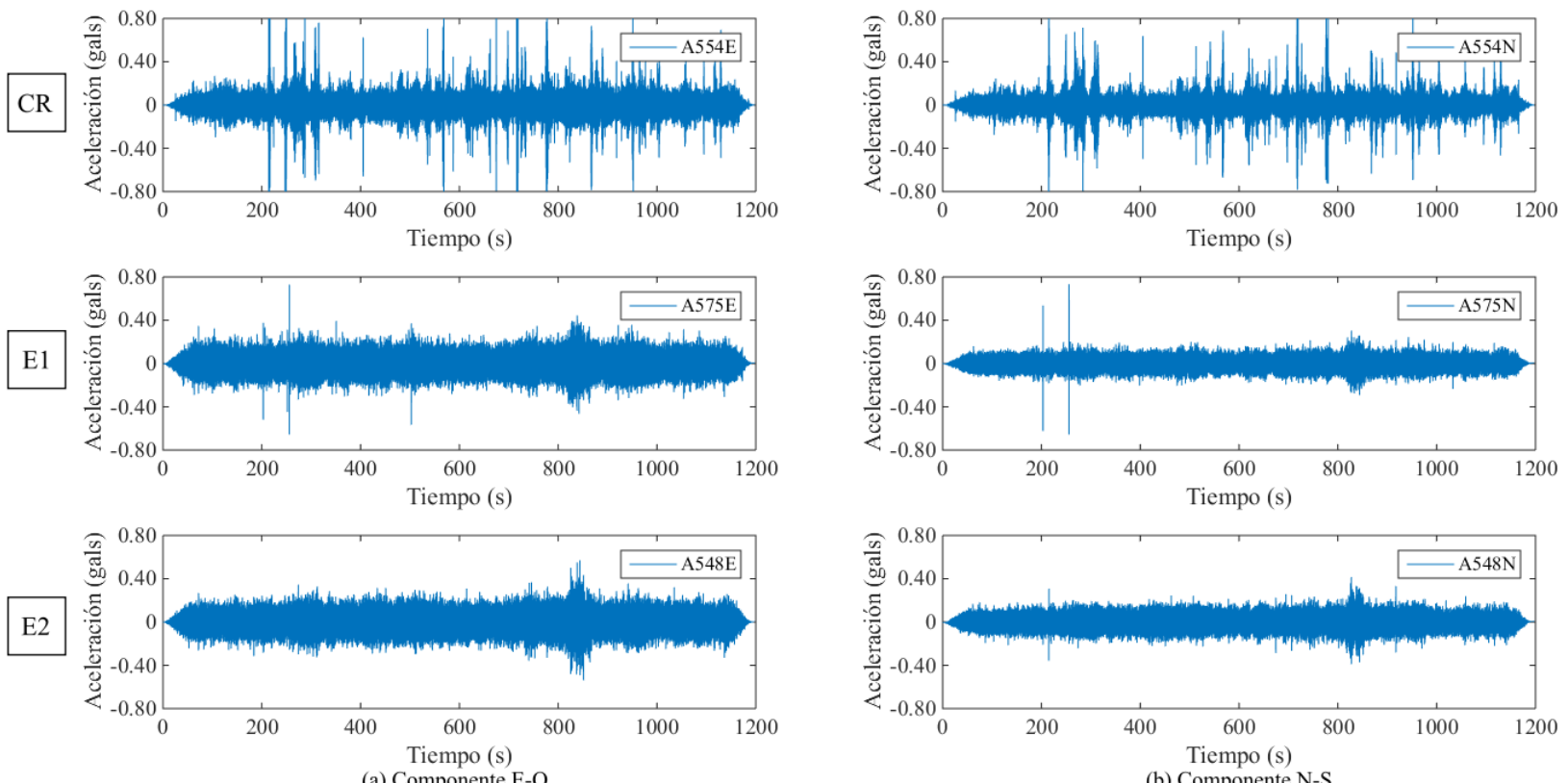

Figura 20. Registros de vibración ambiental para prueba rotacional en nivel N06 del edificio ED-2 

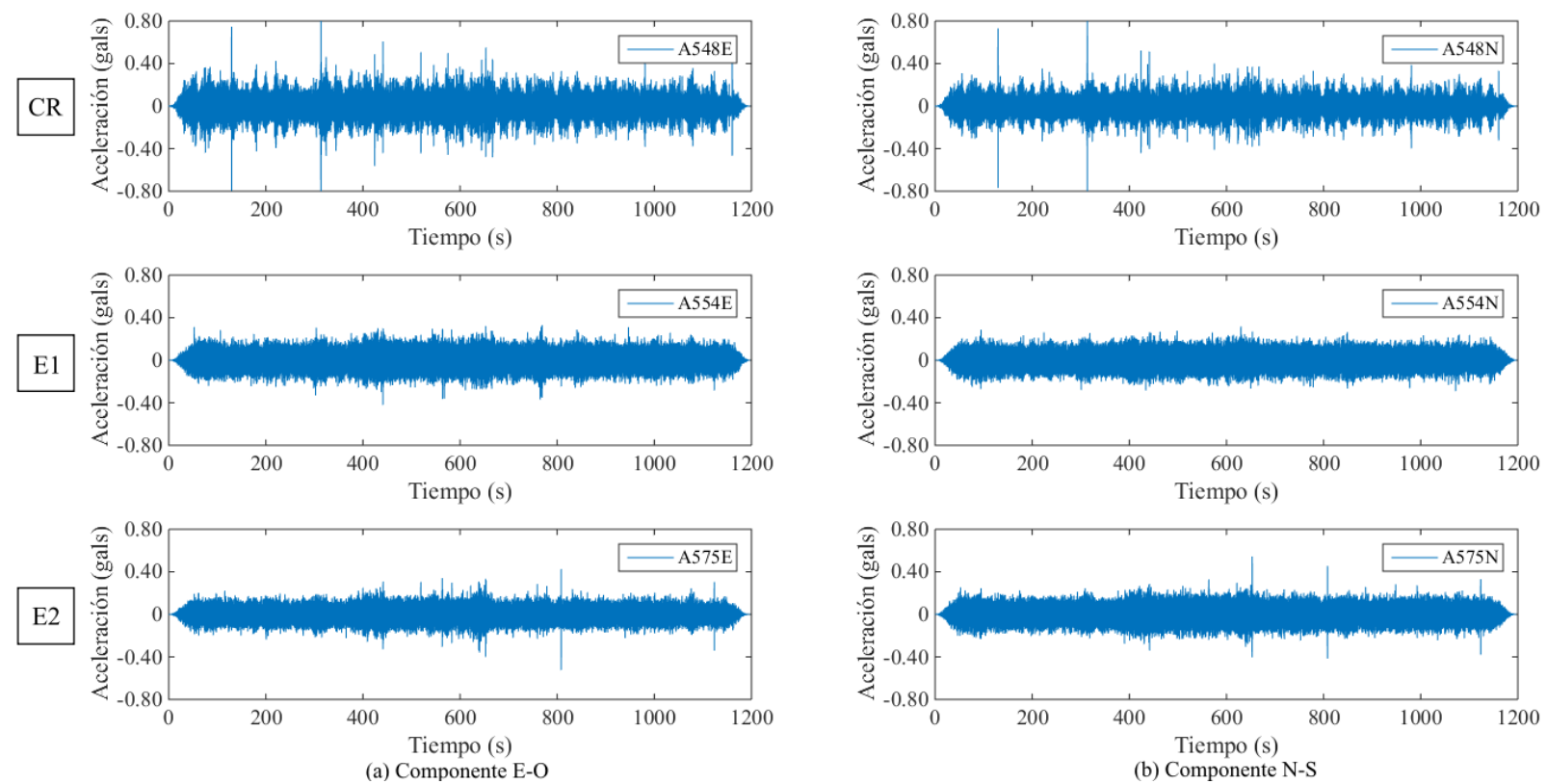

Figura 21. Registros de vibración ambiental para prueba rotacional en nivel N12 del edificio ED-2

Dado que alrededor del acelerómetro situado en las cercanías del centro de rigidez se encuentran los ascensores y las escaleras de la edificación, en los registros de aceleración del mismo se evidencian algunos picos de aceleración en contraste con las mediciones de los instrumentos colocados en los extremos de la planta.

\section{Método básico en el dominio de la frecuencia (BFD)}

A partir de los registros de vibración ambiental de la prueba traslacional, se estimaron las funciones de densidad espectral mostradas en Figura 22. Según estos resultados, la resonancia del sistema estructural ocurre en las frecuencias naturales de $1,025 \mathrm{~Hz}, 1,245 \mathrm{~Hz}, 1,393 \mathrm{~Hz}$ y $2,222 \mathrm{~Hz}$. De las anteriores, solamente en la frecuencia de 2,222 Hz la amplificación ocurre en una única dirección (E-O). No obstante, en las frecuencias de $1,025 \mathrm{~Hz}$ y $1,393 \mathrm{~Hz}$ predomina el movimiento en la dirección E-O, mientras que, en la de $1,245 \mathrm{~Hz}$ la dirección N-S.
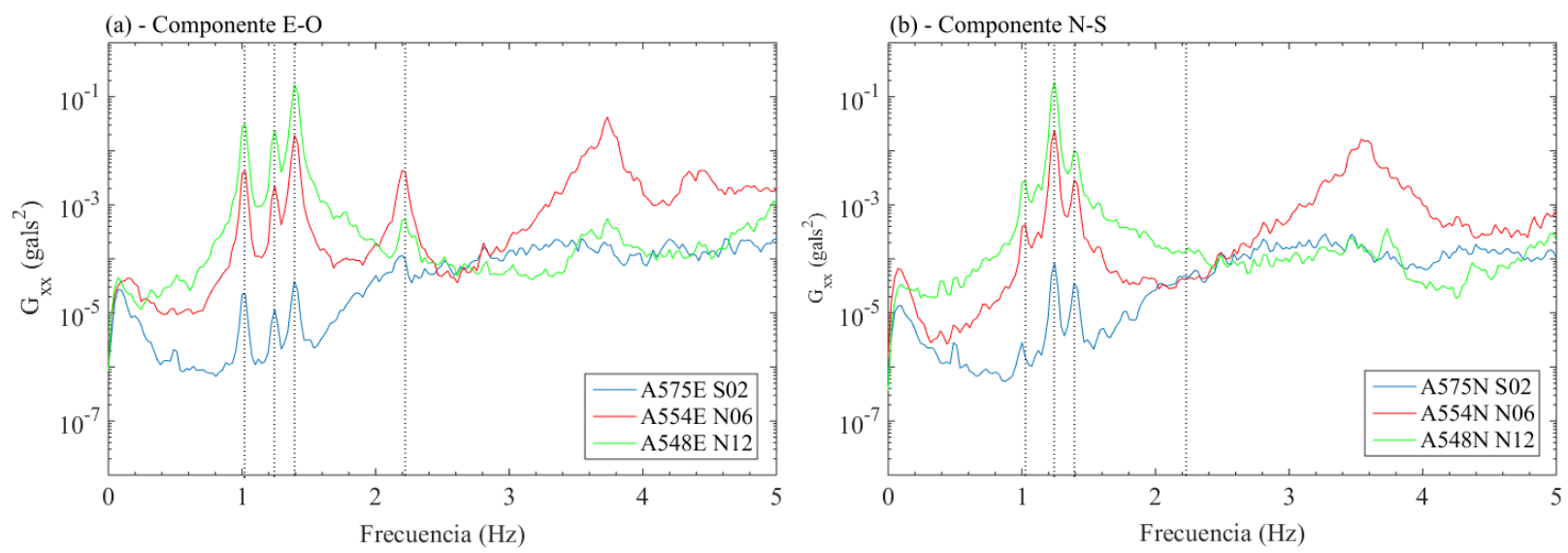

Figura 22. Autoespectros en dirección (a) E-O y (b) N-S de prueba traslacional en edificio ED-2 
Seguidamente, en la Figura 23 se muestran las funciones de coherencia obtenidas al comparar dos sensores ortogonales en un mismo nivel. En este caso, para las frecuencias de $1,025 \mathrm{~Hz}, 1,245 \mathrm{~Hz}$ y 1,393 $\mathrm{Hz}$ se han observado valores de coherencia altos. Debido a lo anterior, se ha considerado que existe un acoplamiento entre modos de flexión.
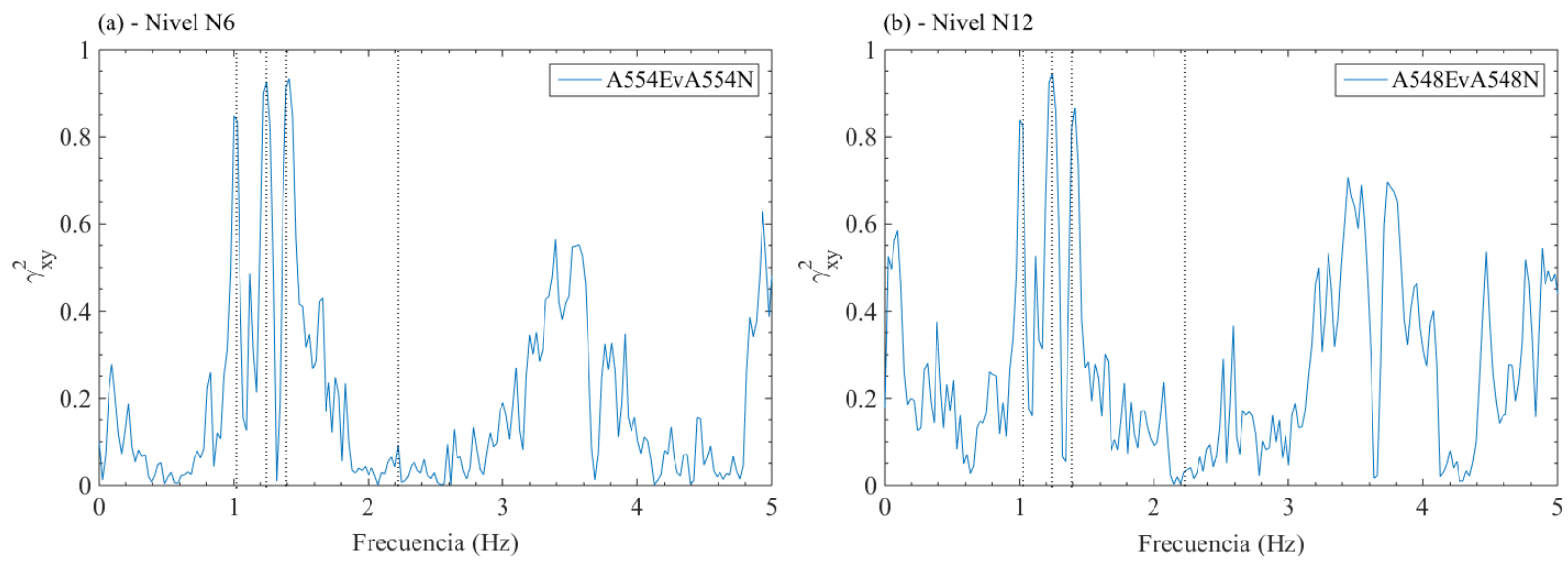

Figura 23. Coherencia entre sensores ortogonales en (a) nivel 6 y (b) nivel 12 en edificio ED-2

Por su parte, en la Figura 24 se presentan los espectros de fase entre dos sensores paralelos ubicados en niveles distintos. Los resultados indican que las señales se encuentran aproximadamente en fase. Sin embargo, debido a la altura de la estructura, esta información no sería suficiente para asegurar que estas frecuencias están asociadas con los primeros modos de flexión de la misma.


Figura 24. Espectro de fase entre sensores paralelos en nivel N6 y N12 en edificio ED-2

Posteriormente, con los registros de vibración ambiental de la prueba rotacional en los niveles N06 y N12, se estimaron los autoespectros mostrados en la Figura 25 y Figura 26, respectivamente. Según los autoespectros, la amplificación del sistema estructural ocurre nuevamente en las frecuencias identificadas de la prueba traslacional. En este caso, se ha determinado que las frecuencias de $1,245 \mathrm{~Hz}$ y 1,393 Hz están asociadas con modos de flexión en las direcciones N-S y E-O, respectivamente. Esto porque las amplitudes entre sensores paralelos resultan ser similares, mientras que, entre sensores ortogonales la diferencia entre amplitudes es significativa. No obstante, en las frecuencias de $1,025 \mathrm{~Hz}$ y $2,222 \mathrm{~Hz}$ se encontró que los sensores en el centro de rigidez presentan amplitudes inferiores en comparación con los sensores en los extremos. Asimismo, los sensores en los extremos presentan amplitudes similares, por lo que estas 
frecuencias pueden estar asociadas con modos de torsión. A pesar de esto, en los autoespectros de las señales paralelas sustraídas (Figura 27) se aprecia que existe una contribución de modos de torsión en todas las frecuencias identificadas anteriormente.
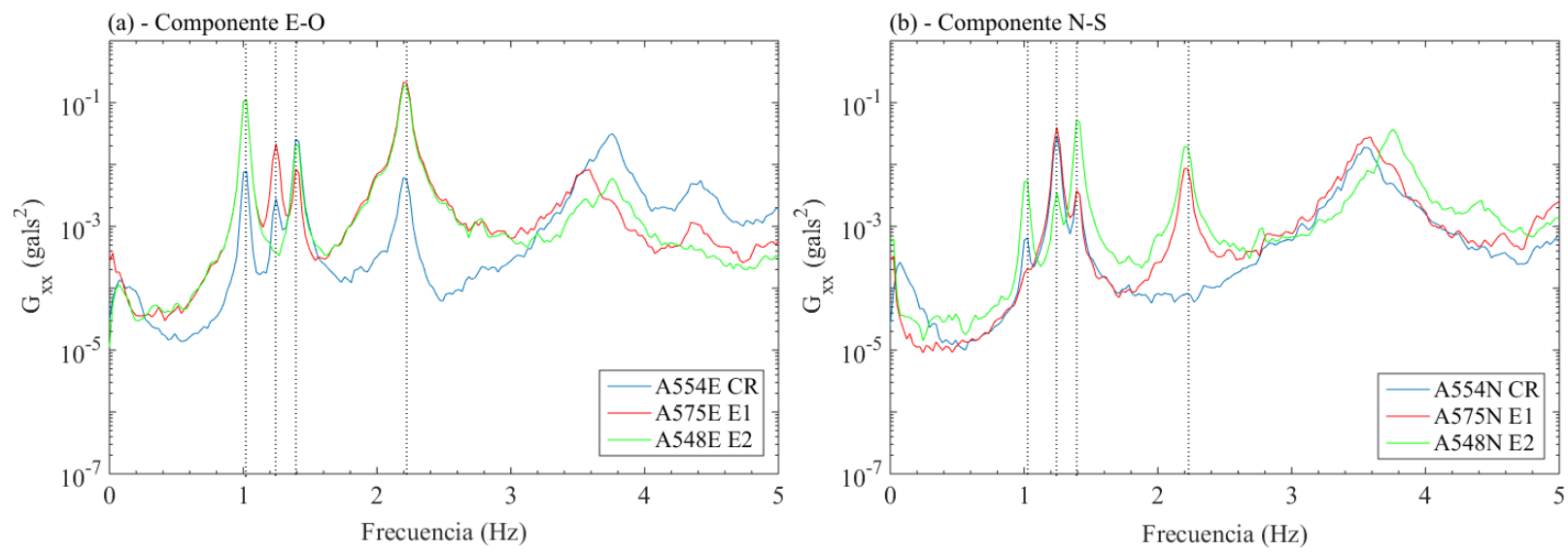

Figura 25. Autoespectros en dirección (a) E-O y (b) N-S de prueba rotacional en nivel N06 del edificio ED-2
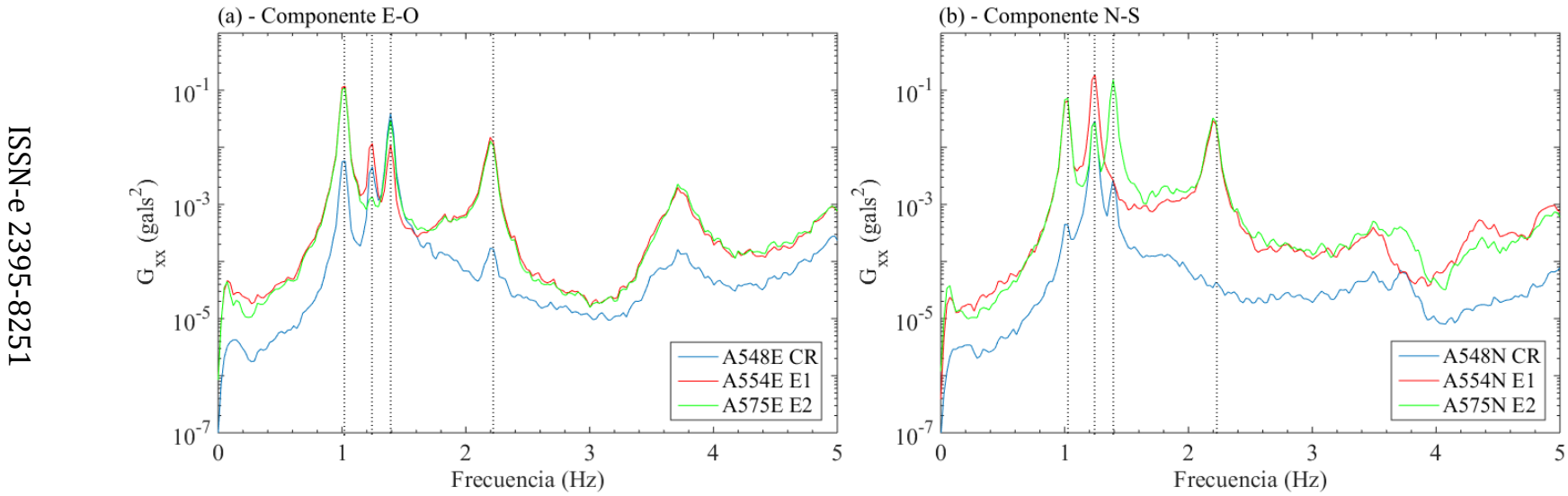

Figura 26. Autoespectros en dirección (a) E-O y (b) N-S de prueba rotacional en nivel N12 del edificio ED-2


Figura 27. Autoespectros de señales sustraídas de la prueba rotacional en niveles (a) N06 y (b) N12 en edificio ED-2 
Con respecto a las funciones de coherencia, se han comparado las señales medidas entre sensores situados en el centro de rigidez y extremos de las plantas de los niveles N06 y N012 (Figura 28 y Figura 29). De acuerdo con los resultados obtenidos, para las frecuencias de $1,025 \mathrm{~Hz}$ y $2,222 \mathrm{~Hz}$ se evidencia alta coherencia entre sensores paralelos en los extremos en ambas direcciones, y en la dirección E-O entre el sensor en el centro de rigidez y en el extremo. Por este motivo, se puede inferir que para estas frecuencias existe un acoplamiento entre un modo de flexión en la dirección E-O con uno de torsión. No obstante, para las frecuencias de $1,245 \mathrm{~Hz}$ y $1,393 \mathrm{~Hz}$ los resultados no son suficientes para distinguir entre el tipo de modo presentado.
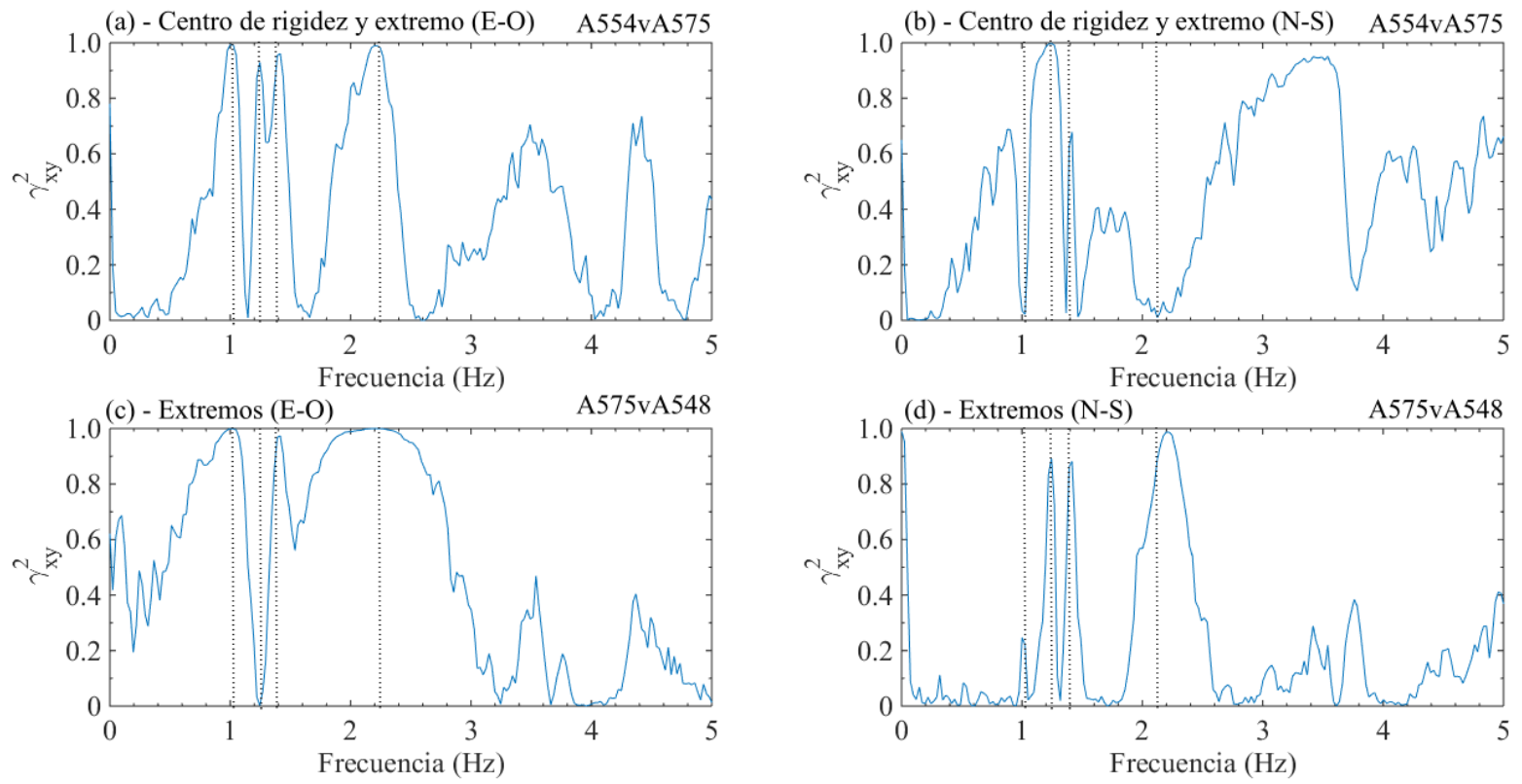

Figura 28. Coherencia entre centro de rigidez y un extremo ( $a$ y b) y extremos ( $c$ y d) en nivel N06 del edificio ED-2
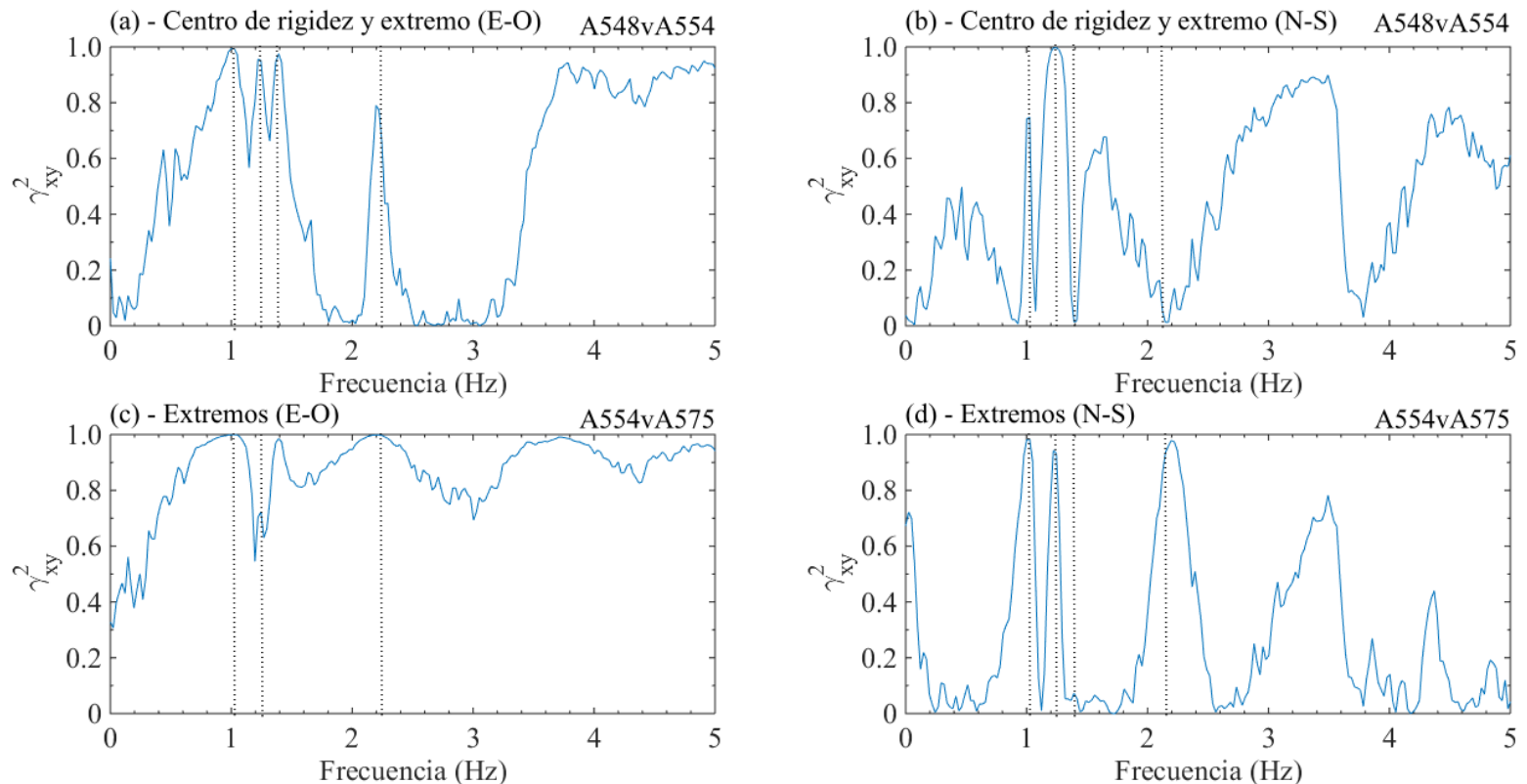

Figura 29. Coherencia entre centro de rigidez y un extremo ( $a$ y b) y extremos (c y d) en nivel N12 del edificio ED-2 
Dado que el método básico la información que suministra corresponde a la deformada operacional, los espectros de fase resultan ser difíciles de interpretar, especialmente en los casos de las estructuras irregulares en planta. Por este motivo, para el edificio ED-2 estos resultados no son presentados.

\section{Método de descomposición en el dominio de la frecuencia (FDD)}

A continuación, los resultados obtenidos de la ejecución del método FDD sobre las funciones de densidad espectral de las pruebas traslacionales y rotacionales del edificio ED-2. Según el primer valor singular (Figura 30), la resonancia del sistema estructural ocurre en las frecuencias de 1,025 Hz, 1,245 Hz, $1,393 \mathrm{~Hz}$ y 2,222 Hz. Es esperable que éstas coincidan con las identificadas con el método BFD, pues los valores singulares, permiten resumir en una sola función todas las funciones de densidad espectral.

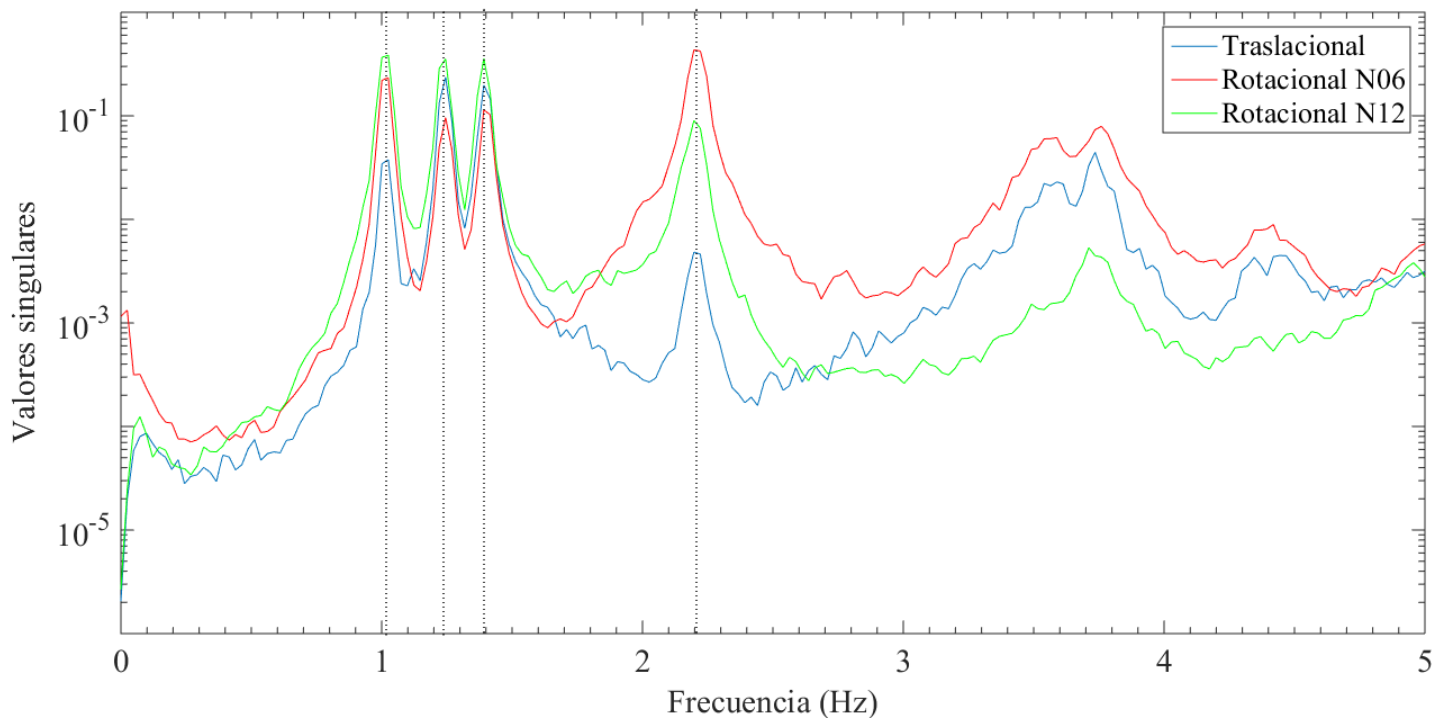

Figura 30. Primer valor singular obtenidos mediante el método FDD en el edificio ED-2

Según la función del primer valor singular, se aprecia que en las frecuencias de $1,025 \mathrm{~Hz}$ y 2,222 Hz la amplitud en la prueba traslacional es significativamente más baja que las de las pruebas rotacionales en los niveles N06 y N12. No obstante, en las frecuencias de 1,245 Hz y 1,393 Hz, la amplitud en la prueba traslacional resulta considerablemente alta. De acuerdo con lo anterior, se espera que en el primer par de frecuencias mencionado esté asociado con modos de torsión, mientras que, el otro par de frecuencias con modos de flexión.

Para la determinación de los tipos de modos de vibración se han usado los vectores singulares mostrados en la Figura 31. En las frecuencias de $1,025 \mathrm{~Hz}$ y $2,222 \mathrm{~Hz}$ (Figura 31 (a) y (d)) se ha encontrado que, para las pruebas rotacionales, en la dirección N-S los sensores situados en los extremos tienen un desfase de aproximadamente $180^{\circ}$ y la amplitud del sensor en el centro de rigidez es baja en comparación con la de los anteriores. No obstante, dado que en la dirección E-O los sensores extremos se encuentran en fase, se considera que sí existe un acoplamiento con un modo de flexión en dicha dirección.

Asimismo, se ha determinado que las frecuencias de 1,245 Hz y 1,393 Hz (Figura 31 (b) y (c)) corresponden a los modos en flexión en la dirección N-S y E-O, respectivamente. Lo anterior porque en cada planta los tres sensores se encuentran aproximadamente en fase para cada dirección predominante. Sin embargo, como las amplitudes no son idénticas para los sensores paralelos, se considera que existe un acoplamiento con otros modos de flexión y torsión. 
Identificación de modos de torsión empleando la medición de vibraciones ambientales y el análisis espectral en dos edificios de concreto reforzado


$$
\begin{array}{llllll}
\text { Simbología } & \text { Traslacional: } & \text { E-O } \bigcirc & \text { S02 } * \text { N06 } \square \text { N12 } \\
& & \text { N-S } \bigcirc & \text { S02 } * \text { N06 } \square \text { N12 } &
\end{array}
$$

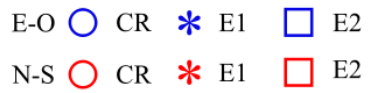

Figura 31. Vectores singulares de las frecuencias naturales identificadas en edificio ED-2 


\section{Formas modales}

De acuerdo con los resultados obtenidos de los métodos BFD y FDD se identificaron dos modos de flexión y dos de torsión. Las formas modales pueden ser observadas en la Figura 32.

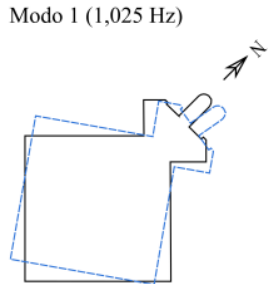

(a) Nivel N06

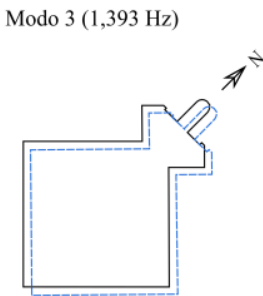

(a) Nivel N06

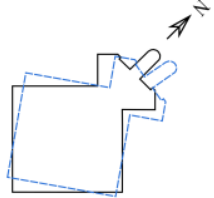

(b) Nivel N12

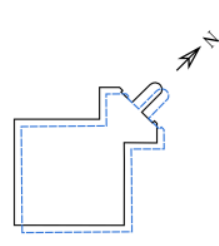

(b) Nivel N12

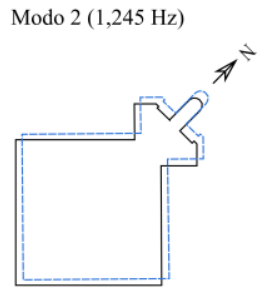

(a) Nivel N06

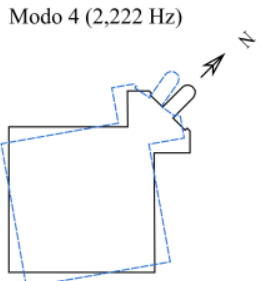

(a) Nivel N06



(b) Nivel N12

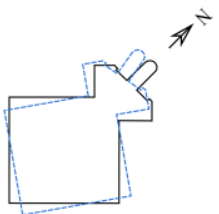

(b) Nivel N12

Figura 32. Formas modales identificadas para edificio ED-2

\section{CONCLUSIONES}

De acuerdo con los resultados experimentales, se identificaron tres modos de vibración para el edificio ED-1, mientras que para el ED-2, cuatro modos de vibración. En este caso, los modos se manifiestan en una banda de frecuencia comprendida entre $1,0 \mathrm{~Hz}$ y $6,0 \mathrm{~Hz}$. Debido a la diferencia de alturas entre los edificios, los modos de vibración del edificio ED-2 ocurren en frecuencias de menor valor que las del ED1. Este resultado coincide con lo esperado dado a que la flexibilidad del sistema estructural tiende a incrementar con respecto a la altura.

Con respecto a la regularidad en la planta, se ha determinado que en el edificio ED-2 existen varios modos de vibración acoplados y que los modos de torsión tienen una contribución significativa en la respuesta del sistema estructural. En esta edificación se encontró que el primer modo de vibración corresponde a uno de torsión. De manera contraria, en el edificio ED-1, donde no hay acoplamiento entre modos de vibración, y los primeros dos corresponden a modos de flexión. A partir de lo anterior, se demuestra experimentalmente que en los sistemas estructurales con plantas irregulares los modos de torsión presentan una contribución significativa en la respuesta dinámica de la estructura, y se manifiestan en un mayor número de frecuencias naturales.

Mediante el método BDF la identificación de los modos de vibración resulta ser sencilla en estructuras regulares en planta y de baja altura. Conforme aumenta la irregularidad y/o la altura de la estructura, las frecuencias naturales pueden ser identificadas. Sin embargo, la interpretación de las formas modales se vuelve más complicada debido al acoplamiento entre modos de vibración, pues el resultado obtenido corresponde a la deformada operacional del edificio. Cuando el acoplamiento entre modos ocurre, las 
funciones de coherencia presentan valores altos entre distintos sensores, de manera que no hay forma de distinguir entre modos de torsión y flexión.

El método FDD resulta ser más eficiente y conveniente para el analista, esto porque permite visualizar todas las funciones de densidad espectral por medio de la función del valor singular. De esta manera, permite identificar todas las frecuencias en las que ocurre la resonancia del sistema estructural, mientras que, con el método BFD se deben verificar cada una de las funciones de densidad espectral. Asimismo, los vectores singulares permiten visualizar en un solo gráfico la posición relativa de los distintos grados de libertad instrumentados, lo cual permite que la identificación de las formas modales resulte ser más sencilla de visualizar en comparación con el método BFD.

Mediante las configuraciones establecidas en la prueba traslacional y rotacional y utilizando tres acelerómetros triaxiales se logran capturar la respuesta de la estructura para la determinación de las frecuencias naturales y de las formas modales. Con esta metodología de instrumentación en aproximadamente una hora y media de duración (incluyendo el tiempo de movilización y colocación de los instrumentos) se logra adquirir toda la información requerida para ejecutar un análisis espectral adecuado en dos edificaciones con una altura total entre $18 \mathrm{~m}$ y $56 \mathrm{~m}$. Esto último resulta ser una estrategia de instrumentación conveniente cuando se dispone de pocos instrumentos, o bien, no se puede instrumentar de forma permanente la estructura.

\section{REFERENCIAS}

Bendat, J y A Piersol (1966). Measurement and Analysis of Random Data. New York: John Wiley \& Sons. Bendat, J y A Piersol (1993). Engineering Applications of Correlation and Spectral Analysis (Second ed.). New York: Jonh Wiley \& Sons.

Bendat, J y A Piersol (2010). Random Data: Analysis and Measurement. New York: John Wiley \& Sons.

Brincker, R y C Ventura (2015). Introduction to Operational Modal Analysis. Chichester: John Wiley \& Sons.

Brincker, R, L Zhang y P Andersen (2001). "Modal identification of output-only systems systems using frequency domain decomposition decomposition". Smart Materials and Structures, 441-445.

Carvajal, O (2005). "Identificación de parámetros dinámicos de dos tipos de edificio utilizando técnicas analíticas y experimentales". Trabajo final de graduación para la obtención del grado de Licenciatura en Ingeniería Civil. San José: Universidad de Costa Rica.

Cherry, S y A G Brady (1959). Determination of Structural Dynamic Properties by Statistical Analysis of Random Vibrations. Shima.

Chopra, A (2007). Dynamic of Structures. New Jersey: Pearson Prentice Hall.

Colegio Federado de Ingenieros y Arquitectos. (2010). Código Sísmico de Costa Rica 2010. San José: Editorial Tecnológica de Costa Rica.

Ditomasso, R, M Vona, M Mucciarelli y A Masi (2010). "Identification of building rotational modes using an ambient vibration technique". European Conference on Engineering Earthquake. Ohrid.

Esquivel, L C y V Schmidt (2016). Mediciones de vibraciones ambientales en tres edificios de concreto reforzado de 28, 11 y 6 pisos. Revista de Ingeniería Sísmica, 81-103. DOI: 10.18867/ris.95.391

Esquivel, L C y V Schmidt (2016). Metodología propuesta para ejecutar y procesar mediciones de vibraciones ambientales utilizando acelerográfos trixales en edificios de concreto reforzado de menos de $100 \mathrm{~m}$ de altura. Revista Internacional de Ingeniería en Estructuras, 21(1), 61-77.

Garita, H (2016). "Análisis modal operacional y calibración del modelo estructural sobre Río Grande en la Ruta Nacional No. 27". Trabajo final de graduación para la obtención del grado de Licenciatura en Ingeniería Civil. San José: UCR. 
Henao, D (2013). "Identificación de propiedades dinámicas de una estructura sometida a vibración ambiental empleando análisis espectral". Proyecto de grado para optar por el título de Magister en Ingeniería. Medellín: Universidad EAFIT.

Irías, M (2016). "Determinación de los parámetros dinámicos de la sección atirantada del puente La Amistad de Taiwán sobre el río Tempisque mediante método de identificación de sistemas multivariables". Trabajo final de graduación para la obtención del grado de Licenciatura en Ingeniería Civil. San José: UCR.

Liu, Y C (2015). Monitoreo estructural de la superestructura No. 2 del puente sobre el Río Virilla Ruta Nacional No. 32. San José: LanammeUCR.

Montalto, E (2016). "Calibración del modelo de elemento finito del puente sobre el río Tenorio en la Ruta Nacional 1 con base en análisis operacional modal". Trabajo final de graduación para la obtención del grado de Licenciatura en Ingeniería Civil. San José: UCR.

Montes, F (2007). Procesos Estocásticos para Ingenieros: Teoría y Aplicaciones. Burjassot: Universitat de València.

Murià, D y R González (1995). "Propiedades dinámicas de edificios de la Ciudad de México". Revista de Ingeniería Sísmica (51), 25-45. DOI: 10.18867/ris.51.261

Peeters, B y G De Roeck (2001). "Stochastic System Identification for Operational Modal Analysis: A Review". Journal of Dynamic Systems, Measurement, and Control, 659-667. DOI: $10.1115 / 1.1410370$

Rainieri, C y G Fabbrocino (2014). Operational Modal Analysis of Civil Engineering Structures: An Introduction and Guide for Applications. New York: Springer.

The Math Works, Inc. (2014). MATLAB (Version 2014b) [Computer Software]. Obtenido de https://www.mathworks.com/

Vandiver, K (2011). The modal expansion theorem. Vibration of Multi-Degree-of-Freedom Systems. Cambridge, Massachusetts: MIT. https://ocw.mit.edu/courses/mechanical-engineering/2-003scengineering-dynamics-fall-2011/vibration-of-multi-degree-of-freedom-systems/the-modalexpansion-theorem/

Yuen, K V, J Beck y L Katafygiotis (2002). Probabilistic approach for modal identification using nonstationary noisy response measurements only. Earthquake Engineering and Structural Dynamics, 1007-1023. DOI: $10.1002 /$ eqe. 135 